\title{
Corrupción, estructura productiva y desarrollo económico en los países en desarrollo
}

\author{
Helis Cristina Zanuto Andrade Santos \\ y Gilberto Joaquim Fraga ${ }^{1}$
}

\section{Resumen}

En los últimos años la corrupción ha alcanzado niveles alarmantes: cada año el costo de la corrupción equivale aproximadamente al 5\% del producto mundial. En este contexto, el objetivo de este trabajo consiste en investigar la manera en que la corrupción y la estructura productiva inciden en el desarrollo socioeconómico de los países en desarrollo. Para alcanzar este objetivo, se utilizó el procedimiento de datos de panel dinámico para el período 2002-2014. Entre los principales resultados, se observa que la corrupción tiene un comportamiento no lineal en relación con el desarrollo. Asimismo, es necesario que el estudio de los distintos aspectos del desarrollo comprenda tanto el punto de vista económico como el social. En general, hay indicios de que una estructura productiva más sofisticada distorsiona los efectos de control de la corrupción en el desarrollo socioeconómico, pues potencia la influencia de la corrupción en dicho desarrollo.

\section{Palabras clave}

Corrupción, corrupción en las empresas, aspectos económicos, producción, productividad, desarrollo económico, desarrollo social, análisis económico, países en desarrollo

\section{Clasificación JEL}

O11, O50, E00

\section{Autores}

Helis Cristina Zanuto Andrade Santos es Doctoranda en Economía de la Industria y Tecnología de la Universidad Federal de Río de Janeiro (Brasil) y Profesora sustituta en el Departamento de Economía de la Universidad Estadual de Maringá (UEM) (Brasil). La autora agradece al Consejo Nacional de Desarrollo Científico y Tecnológico (CNPq) por el apoyo para la elaboración del presente artículo, redactado durante su maestría (2015-2016). Correo electrónico: helis_czas@hotmail.com.

Gilberto Joaquim Fraga es Profesor asociado en el Departamento de Economía de la Universidad Estadual de Maringá (UEM) (Brasil). Correo electrónico: gjfraga@uem.br.

Los autores agradecen a los evaluadores anónimos que comentaron la primera versión de este texto y los eximen de toda responsabilidad en esta versión final. 


\section{Introducción}

La globalización y la rapidez con que se difunde la información han permitido detectar mayores indicios de corrupción, que alimentaron la preocupación de las autoridades y dieron lugar a estudios sobre el tema. Cada año, el costo global de la corrupción representa más del 5\% del producto interno bruto (PIB) mundial, que equivale a 2,6 billones de dólares. De acuerdo con el Foro Económico Mundial (2012), más de 1 billón de dólares se paga en sobornos. En este contexto, muchos investigadores académicos y no académicos analizan tanto los determinantes de la corrupción como sus efectos en el crecimiento y el desarrollo de las economías.

Uno de los primeros estudios relacionados con el tema es el de Leff (1964), que considera que la corrupción puede tener una influencia positiva en el crecimiento. La hipótesis es que la corrupción actúa como "lubricante de las ruedas del crecimiento económico". Los trabajos más recientes se centran en el gasto en tecnologías sofisticadas en países que no necesitarían ese estándar y en la sofisticación de la estructura productiva, que se han sugerido, entre otros, como posibles factores potenciadores de la corrupción. En este sentido se destacan Shleifer y Vishny (1993) y Rose-Ackerman (1997), que realizaron importantes aportes teóricos al área de estudio que relaciona la corrupción con la sofisticación de la estructura productiva y el desarrollo económico.

El modelo teórico de Shleifer y Vishny (1993) tiene en cuenta la oferta y la demanda de bienes del gobierno por agentes privados, tanto a un precio normal que se ha de cobrar, como a un precio que incluye un soborno. Los autores argumentan que los países pobres prefieren gastar sus recursos en proyectos de infraestructura y defensa, que presentan mayores oportunidades de corrupción, que en mejoras en la educación y la salud. En cuanto a las nuevas tecnologías, estas podrían centrarse en monopolios u oligopolios para garantizar la continuidad de los sobornos y la malversación de fondos. En este caso, existen barreras a la entrada de nuevas empresas y a la innovación, que deterioran las inversiones y el crecimiento de la economía. Rose-Ackerman (1997) argumenta que la corrupción puede tener una influencia positiva y que, en algunos casos, los sobornos y las comisiones ilegales pueden no redundar en costos para la sociedad. Sin embargo, una vez que la persona sucumbe a estos, es probable que se corrompa cada vez más, hasta que se produzcan efectos negativos.

Si bien en la literatura existen diversos estudios que tratan de explicar el desarrollo, se observa una laguna en cuanto a los efectos concomitantes de la estructura productiva y la corrupción en el mejoramiento del nivel de vida de las naciones. En vista de ello, el análisis de los efectos simultáneos de la estructura productiva del país y de la corrupción en el desarrollo cobra relevancia.

En este contexto, el objetivo de la presente investigación consiste en analizar la relación entre la corrupción, la estructura productiva y el desarrollo. Además, se examinan los posibles efectos de la interacción de la corrupción con la estructura productiva del país en el desarrollo socioeconómico de los países en desarrollo.

Como medida del desarrollo social se utiliza el porcentaje de la población subalimentada y, para el desarrollo económico, el logaritmo del producto per cápita. La corrupción está representada por el indicador de control de la corrupción. Los productos sectoriales de la industria, la agricultura y los servicios y el índice de complejidad económica representan la estructura productiva. En la estimación empírica se controlan otros efectos, como el nivel de capital humano, la inversión extranjera y el agua potable. Las estimaciones se realizan mediante el procedimiento de datos de panel dinámico.

El artículo se divide en cinco secciones, incluida esta introducción. Mientras en la segunda sección se presenta la literatura empírica, en la tercera se describen los datos y la estrategia empírica utilizados y en la cuarta se detallan y analizan las estimaciones econométricas. En la quinta y última sección se realizan las consideraciones finales. 


\section{Literatura empírica}

En esta sección se presentan algunos estudios empíricos en los que se aborda la relación entre la corrupción, la estructura productiva y el desarrollo. Entre los autores que examinan los efectos de la corrupción en el desarrollo se encuentran Akçay (2006), Sodré (2014) y Dalberto (2016). Estos autores encontraron resultados econométricos que sugieren una relación negativa entre la corrupción y el desarrollo o el crecimiento. Algunos resultados apuntan incluso a que la corrupción aumentaría la concentración y la desigualdad de ingresos e incrementaría la proporción de pobres.

Otras investigaciones sugieren que la representación de los efectos de la corrupción presenta forma de U o no lineal. Li, Xu y Zou (2000) encontraron que, con un nivel de corrupción alto o bajo, la desigualdad de ingresos es menor, mientras un nivel de corrupción medio está asociado con grandes desigualdades de ingresos. Houston (2007) realiza un análisis exhaustivo de los efectos de la corrupción en el crecimiento de la economía para verificar si son expansionistas o restrictivos y concluye que pueden ser de ambos tipos, según el grado de aplicación de las leyes de protección de la propiedad del país. Aidt, Dutta y Sena (2008) estudian los efectos no lineales de esa relación y las diferencias en la calidad institucional. En los países con instituciones políticas de alta calidad, la corrupción tiene un efecto negativo en el crecimiento, mientras en los países donde la calidad de las instituciones es baja, la corrupción no tiene efectos en el producto. Esto remite a la idea de corrupción como "lubricante de las ruedas del crecimiento económico".

En el análisis de Swaleheen (2011) se indica que la corrupción tiene un efecto directo e indirecto en el crecimiento, que depende del nivel de corrupción del país en cuestión. En esa misma línea, Sobral (2014) explica que la relación entre las variables puede no ser lineal en el caso de los países con mayor libertad civil.

En los estudios se utilizan diferentes criterios para medir los efectos de la estructura productiva en el desarrollo. Por ejemplo, Hartmann y otros (2015) utilizan el índice de complejidad económica como variable sustitutiva de la sofisticación de la producción y el coeficiente de Gini del producto. Los autores encuentran que, además de contribuir al crecimiento económico, el aumento de la complejidad económica está acompañado por una reducción de la desigualdad de ingresos.

Mauro (1997b, 1998) encuentra que a los gobiernos corruptos les resulta más fácil cobrar sobornos en algunos tipos de gastos que en otros. Los grandes proyectos de difícil valoración, como los proyectos de infraestructura o los sistemas de defensa de alta tecnología, serían más propicios para la corrupción que los salarios de los profesores, por ejemplo, porque cambiaría la composición del gasto público (reduciendo los gastos en educación). Ruhashyankiko y Yehoue (2006) sugieren que la expansión de los sectores privados inducida por la tecnología se traduce en una disminución de la corrupción agregada y que la disminución de la corrupción pública superaría un posible aumento de la corrupción privada.

En el estudio de Mahdavi (2014) se afirma que existe una relación entre la corrupción y los países con una estructura orientada a la producción de petróleo solamente cuando los países establecen agencias nacionales de regulación del petróleo. Esas agencias pueden facilitar la corrupción, pues se establecen con indicaciones políticas, y sus funcionarios tienen mayores oportunidades de solicitar sobornos. Además de la facilidad por las conexiones políticas, hay también una mayor propensión a la corrupción, pues esos gestores tienen las licencias de petróleo y el acceso a los contratos, y pueden incrementar su rentabilidad.

A partir de un modelo de crecimiento, Leite y Weidmann (1999) analizan las relaciones entre recursos naturales, corrupción y crecimiento económico. Los autores encuentran que los efectos negativos de la corrupción en el crecimiento económico tienen lugar de manera no lineal: los efectos se intensifican en los países menos desarrollados y la abundancia de recursos naturales crea oportunidades para actividades de captación de rentas, que contribuyen al nivel de corrupción. 
Por último, en un estudio de la Organización de Desarrollo y Cooperación Económicos (OCDE, 2013) se exponen algunos casos particulares de países que presentan un alto crecimiento económico en ambientes que no son favorables para ello². A medida que países como la República de Corea y zonas como Taipei (China) pasan al grupo de altos ingresos, registran mejoras en sus niveles de corrupción. Esta situación puede indicar que, si bien los niveles de corrupción permanecían altos mientras esas economías alcanzaban mayores niveles tecnológicos, la estructura productiva puede haber atenuado los efectos perversos de la corrupción en ese período, visto que lograron alcanzar un alto crecimiento en ese ambiente.

\section{Datos y estrategia empírica}

\section{Base de datos y descripción de las variables}

Para alcanzar los objetivos planteados, se utilizaron datos organizados en forma de panel correspondientes al período 2002-2014 y relativos a 98 países en desarrollo, que se detallan en el anexo A1.

Se seleccionaron dos variables sustitutivas para la estructura productiva y una para la corrupción. La sofisticación de la estructura productiva estará representada por el índice de complejidad económica (ECl, por sus siglas en inglés) y por las partes correspondientes al PIB industrial, agropecuario y de servicios ${ }^{3}$. La corrupción estará representada por el indicador de control de la corrupción (CC), basado en la solidez de la gobernanza del país ${ }^{4}$. A continuación se presenta la interacción del indicador CC con cada una de las variables sustitutivas de la estructura productiva para probar la significación de cada resultado.

Conforme la literatura empírica, la definición e interpretación de las variables es la siguiente ${ }^{5}$ :

i) loggdp: logaritmo del "PIB per cápita (en dólares constantes de 2010)" - cuanto mayor sea el valor de loggdp, mayor desarrollo se espera;

ii) sub: "población subalimentada (en porcentajes)", que está por debajo del nivel mínimo de consumo de energía alimentaria (Naciones Unidas, s/fa y s/fb) ${ }^{6}$ - cuanto mayor sea el valor de sub, menor desarrollo se espera;

iii) eci: "índice de complejidad económica", que representa la complejidad de los productos del país (OEC, s/fa y s/fc) - cuanto mayor sea el valor de eci, mayor sofisticación productiva, mayor loggdp y menor sub se espera;

2 Rock y Bonnett (2004) explican que la corrupción reduce el crecimiento en la mayoría de los países en desarrollo, sobre todo en los países pequeños, pero aumenta el crecimiento en los países más grandes de Asia Oriental de reciente industrialización.

3 Si bien la variable ECl se utiliza para representar el conocimiento que tiene un determinado país, se construye conforme la ubicuidad y la diversidad de los productos que este exporta. La variable representará una economía más compleja o con estructura productiva sofisticada en términos de conocimiento. Véase más información en el Atlas elaborado por Hausmann y otros (2011). Al considerar los PIB sectoriales, la sofisticación de la estructura productiva se entenderá en el sentido tecnológico o de maquinaria.

4 Indicador de percepción de cuán controlada ha estado la corrupción en el país. Cuanto menor sea su valor, menor será el control de la corrupción del país, es decir, mayor será la percepción de corrupción. Se construye a partir de la percepción del poder público ejercido para beneficio privado, incluidas las pequeñas y grandes formas de corrupción y los intereses de las élites (Kaufmann, Kraay y Mastruzzi, 2010; Banco Mundial, s/fc).

5 En algunas estimaciones se utilizaron las variables "Crédito interno proporcionado por el sector financiero (como porcentaje del PIB)" y "Exportaciones de alta tecnología (como porcentaje de las exportaciones de manufacturas)" del Banco Mundial (s/fa), fuente también de las variables de los ítems i), iv), v), vi), viii), ix) y xii).

6 Para su cálculo se utiliza el nivel mínimo de energía alimentaria, que difiere según el género, la edad y los diferentes niveles de actividad física (FAO, 2016). De acuerdo con datos disponibles en FAO (2016) sobre diversos países, en el período 2014-2016 el requisito mínimo de energía alimentaria varió entre 1.654 kilocalorías por persona por día para Timor-Leste y 1.987 kilocalorías por persona por día para los Emiratos Árabes Unidos. Los valores correspondientes a los "países menos desarrollados" y al "mundo" fueron, respectivamente, 1.747 y 1.844 kilocalorías por persona por día. 
iv) gdpagro: "agricultura, valor agregado (como porcentaje del PIB)" - cuanto mayor sea el valor de gdpagro, menor sofisticación productiva con respecto a la parte del PIB de la industria y menor sub se espera;

v) gdpind: "industria, valor agregado (como porcentaje del PIB)"- cuanto mayor sea el valor de gdpind, mayor sofisticación productiva con respecto a las partes del PIB de la agricultura y de los servicios, mayor loggdp y mayor sub se espera;

vi) gdpser: "servicios y otros, valor agregado (como porcentaje del PIB)"- cuanto mayor sea el valor de gdpser, menor sofisticación productiva con respecto a la parte del PIB de la industria, mayor loggdp y menor sub se espera;

vii) controlcorrup: "control de la corrupción" (Banco Mundial, s/fb y s/fc) - cuanto mayor sea el valor de controlcorrup, menor corrupción, mayor loggdp y menor sub se espera;

viii) abecom: "comercio (como porcentaje del PIB)" - cuanto mayor sea el valor de abecom, mayor apertura comercial, mayor loggdp y menor sub se espera;

ix) fdii: "inversión extranjera directa, entradas netas (como porcentaje del PIB)" - cuanto mayor sea el valor de fdii, mayor entrada neta de inversión extranjera directa, mayor loggdp y menor sub se espera;

x) agua: "proporción de la población que utiliza fuentes de agua potable mejoradas, total" (Naciones Unidas, s/fa) - cuanto mayor sea el valor de agua, mayor proporción de la población que utilice fuentes de agua potable mejoradas, mayor loggdp y menor sub se espera;

xi) hc: "índice de capital humano, basado en los años de estudio y el retorno a la educación" (Feenstra, Inklaar y Timmer, 2015) - cuanto mayor sea el valor de hc, mayor índice de capital humano, mayor loggdp y menor sub se espera;

xii) gini: "coeficiente de Gini" - cuanto mayor sea el valor de gini, mayor desigualdad de ingresos y mayor sub se espera;

xiii) ores_metal: "minerales y metales (como porcentaje de la participación de productos de exportación" (WITS, 2018) - cuanto mayor sea el valor de ores_metal, mayor abundancia de productos básicos no agrícolas, mayor loggdp y mayor sub se espera.

Las estadísticas descriptivas de las variables se detallan en el cuadro 1.

Los valores medios de las variables sub y loggdp para la muestra de países seleccionada son, respectivamente, 14,997 y 8,033, mientras controlcorrup tiene una media de 0,361 . Para la estructura productiva, eci, gdpind, gdpagro y gdpser presentan valores medios de -0,366, 32,576, 15,253 y 52,156 , respectivamente. Cuando estas variables interactúan con controlcorrup, los valores medios de sus interacciones pasan, respectivamente, a -0,103, 11,740, 5,066 y 19,508.

En 2014, el porcentaje de población subalimentada (Naciones Unidas, s/fa) fue elevado en muchos países de África y Centroamérica. De estos países en desarrollo, Haití registró el mayor porcentaje de población subalimentada (el 52,3\% de su población se situaba por debajo del nivel mínimo de consumo de energía alimentaria).

Los datos de crecimiento del PIB per cápita (Banco Mundial, s/fa) por regiones muestran que, en África y Centroamérica, los datos de subalimentación coinciden con un bajo PIB per cápita en 2014. En términos generales, Asia Meridional parece diferir bastante al comparar esos indicadores, pues esta región presenta la mayor tendencia de crecimiento del PIB per cápita (un 5,4\% en 2014) y, al mismo tiempo, porcentajes elevados de población subalimentada. Las diferencias que se pueden observar al comparar los indicadores socioeconómicos de desarrollo obedecen a distintos factores, como la metodología de cálculo del indicador o las distintas características de los países. Es probable que las características de un país de Asia Meridional difieran bastante con respecto a otro país del mismo grupo y que estas sean captadas por un indicador y no por otro. 
Cuadro 1

Estadísticas descriptivas, 2002-2014

\begin{tabular}{|c|c|c|c|c|c|}
\hline Variable (global) & Media & $\begin{array}{l}\text { Desviación } \\
\text { estándar }\end{array}$ & Mínimo & Máximo & Observaciones \\
\hline sub & 14,99677 & 11,20825 & 5 & 53,5 & $N=1144$ \\
\hline $\log d p$ & 8,032544 & 1,330887 & 5,268729 & 11,22033 & $N=1272$ \\
\hline controlcorrup & 0,3610675 & 0,1279468 & 0 & 0,75 & $N=1274$ \\
\hline eci & $-0,3656327$ & 0,76884 & $-3,17674$ & 1,86034 & $N=1074$ \\
\hline gdpind & 32,57637 & 13,80958 & 6,896044 & 77,41366 & $N=1173$ \\
\hline gdpagro & 15,25261 & 12,21122 & 0,0344685 & 58,20515 & $N=1175$ \\
\hline gdpser & 52,15547 & 12,53512 & 18,90939 & 92,98227 & $N=1171$ \\
\hline corceci & $-0,1025409$ & 0,3013437 & $-0,9692$ & 1,395255 & $N=1074$ \\
\hline corind & 11,73966 & 6,391206 & 0 & 45,2799 & $N=1173$ \\
\hline coragro & 5,066135 & 4,306053 & 0 & 27,06063 & $N=1175$ \\
\hline corser & 19,50751 & 10,37118 & 0 & 69,7367 & $N=1171$ \\
\hline abecom & 87,028 & 57,6045 & 19,11879 & 455,2767 & $N=1240$ \\
\hline fdii & 4,817579 & 7,002263 & $-8,400837$ & 89,47596 & $N=1256$ \\
\hline cre & 47,5557 & 42,93937 & $-114,6937$ & 236,1799 & $N=1231$ \\
\hline tech & 8,173824 & 12,39399 & 0 & 74,17846 & $N=1098$ \\
\hline agua & 82,90496 & 16,30681 & 33 & 100 & $N=1231$ \\
\hline hc & 2,234161 & 0,5793039 & 1,088122 & 3,593633 & $N=1105$ \\
\hline gini & 43,10034 & 9,424866 & 24 & 59,5 & $N=291$ \\
\hline ores_metal & 9,430601 & 15,58277 & 0 & 86,42 & $N=1131$ \\
\hline corcon & 0,1467273 & 0,1076587 & 0 & 0,5625 & $N=1274$ \\
\hline
\end{tabular}

Fuente: Elaboración propia.

Nota: En el caso de algunas variables no se dispone de información sobre todos los países. Para la variable gini, existen 24 países: Argentina, Armenia, Belarús, Bolivia (Estado Plurinacional de), Brasil, Colombia, Costa Rica, Ecuador, El Salvador, Federación de Rusia, Georgia, Honduras, Kazajstán, México, Panamá, Paraguay, Perú, República de Moldova, República Dominicana, Serbia, Tailandia, Turquía, Ucrania, Uruguay. Para las otras variables, faltan los siguientes países: variable sub, no incluye: Bahamas, Bahrein, Burundi, Hong Kong (RAE de China), Libia, Papua Nueva Guinea, Qatar, República Democrática del Congo, Singapur y Sudán; variables eci y corceci, Bahamas, Brunei Darussalam, Burundi, Gambia, Guinea-Bissau, Guyana, República Democrática del Congo, Sierra Leona, Suriname; variables gdpind, gdpagro, gdpser, corind, coragro y corser, Angola, Bahrein, Kuwait, Liberia, Libia; variable fdii, Cuba; variable cre, Cuba, Irán; variable tech, Angola, Guinea-Bissau, Liberia, Libia, República Democrática del Congo; variable agua, Brunei Darussalam, Hong Kong (RAE de China), Libia; variable hc, Azerbaiyán, Bahamas, Belarús, Cuba, Georgia, Guinea, Guinea-Bissau, Guyana, Líbano, Libia, Omán, Papua Nueva Guinea, Suriname; variable ores_metal, Liberia, República Democrática del Congo.

En vista de ello, el análisis de la corrupción y la estructura productiva puede contribuir a una mejor comprensión de las características de esos países y de los valores de sus indicadores de desarrollo. En 2014, los menores niveles de control de la corrupción parecían prevalecer en América Latina y el Caribe, África y algunas áreas de Asia. El Sudán fue el país con menor control de la corrupción, mientras Suecia se situó entre los menos corruptos (Banco Mundial, s/fb).

En el gráfico 1 se muestra la relación entre el índice de complejidad económica y el PIB per cápita en 2014. Si bien los países que tienen una gran producción con respecto al número de habitantes presentan altos niveles de PIB per cápita, dicha producción puede estar concentrada en pocos habitantes. Así, al analizarse individualmente, el PIB per cápita puede ocultar el nivel de vida real del país; de ahí la importancia de considerar también el porcentaje de subalimentación.

En 2014, el Japón ocupó el primer lugar en la clasificación de países con mayor complejidad económica en 2014, mientras Sudán del Sur se situó en el último (OEC, s/fa). 


\section{Gráfico 1}

Países en desarrollo seleccionados: comparación del índice de complejidad económica y el PIB per cápita, 2014

(En dólares constantes de 2010)

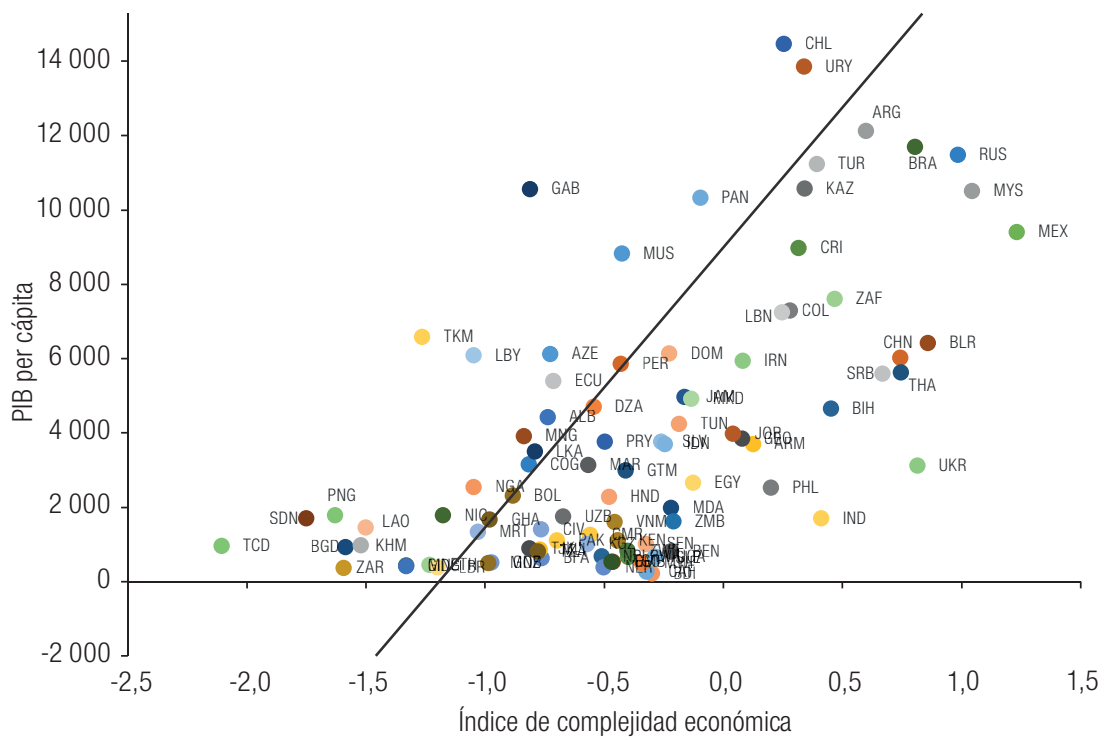

Fuente: Elaboración propia, sobre la base de Observatorio de Complejidad Económica (OEC), "Economic complexity rankings", Cambridge, Massachusetts Institute of Technology (MIT), s/fa [en línea] https://oec.world/en/rankings/country/eci/.

Nota: Los datos se refieren a los 98 países en desarrollo que se detallan en el anexo A1.

Después de esta breve exposición y comparación de algunos indicadores, se verifica la necesidad de diferentes variables sustitutivas para poder interpretar el comportamiento de las economías. Así, en las próximas secciones se realizarán estimaciones y pruebas econométricas, centradas en el desarrollo social y en el desarrollo económico.

\section{Estrategia empírica}

Aidt, Dutta y Sena (2008) analizan el papel de la responsabilidad política como determinante de la corrupción y del crecimiento económico. Al tratar la corrupción como una variable endógena y no lineal relacionada con las diferencias en la calidad institucional, los autores encuentran que la relación entre la corrupción y el crecimiento es específica del régimen de gobernanza. Sus aportes empíricos son: el modo de elegir los regímenes para los diferentes conjuntos de países; la propuesta de un modelo que permite efectos de umbral y obtener resultados relativos a las consecuencias de la corrupción en el crecimiento; la estimación del impacto del crecimiento en la corrupción; la introducción de no linealidades en la relación corrupción-crecimiento; la diferenciación de tasas de crecimiento a corto y largo plazo y el uso de variables instrumentales. Se argumenta que los vínculos entre la corrupción, el crecimiento y las instituciones pueden no ser sencillos y que, por lo tanto, deben considerarse los efectos no lineales y de causalidad inversa.

En este sentido, se propone un modelo empírico de panel dinámico inspirado en Aidt, Dutta y Sena (2008). La corrupción se tratará como una variable de interacción con la estructura productiva (puede haber causalidad inversa) y se prestará atención a las diferencias de estructura que puedan existir. La metodología elegida (método generalizado de momentos) permitirá el uso de variables instrumentales. Posteriormente, también se considerarán los posibles efectos de no linealidad. El modelo empírico que se ha de estimar se presenta en la ecuación (1):

$$
D E_{i t}=\alpha_{0}+\lambda D E_{i t-1}+\beta_{1} \sum_{i=1}^{3} E P_{i t}+\beta_{2} C_{i t}+\beta_{3} C \sum_{i=1}^{3} E P_{i t}+\beta_{4} V C_{i t}+\mu
$$


El desarrollo (DE) será la variable que se ha de explicar ante sus efectos rezagados, las variables de control (VC), las variables de interés seleccionadas y el error ( $\mu$ ). La medida del desarrollo se representará desde el punto de vista económico, mediante (loggdp), y social, mediante (sub). El desarrollo del país presenta una transición lenta a lo largo del tiempo y el desarrollo pasado influye en la etapa de desarrollo actual. Debido a que su evolución depende de características institucionales y sociales que no cambian con frecuencia, el uso del panel dinámico resulta apropiado. Según el tipo $i$ de estructura productiva (EP) que predomina en el país (agrícola, industrial o de servicios), la relación con el desarrollo puede diferenciarse, al suponer una mayor o menor sofisticación de dicha estructura. La categoría de estructura productiva predominantemente industrial puede representarse por la medida (eci).

Para la corrupción $(C)$ se utilizará el indicador de control de la corrupción (controlcorrup). Se espera que la relación entre este indicador y el desarrollo socioeconómico sea positiva, pues cuanto mayor sea el control de la corrupción, menor será el nivel de corrupción percibida en el país, lo que mejora el desarrollo socioeconómico, como se sugiere en general en la literatura. Por lo tanto, se espera que la relación entre (controlcorrup) y (loggdp) sea positiva, mejorando el desarrollo económico, y que la relación entre (controlcorrup) y (sub) sea negativa, dado que el mayor control de la corrupción y la consecuente mejora del desarrollo social podrían contribuir a reducir la subalimentación.

Con respecto a la variable de interacción entre la corrupción y la estructura productiva $\left(C \sum_{i=1}^{3} E P_{i}\right)$, se sugiere una causalidad inversa de dependencia entre ellas. Por una parte, las personas estarían dispuestas a optar por tecnologías más avanzadas de lo necesario, debido a la mayor facilidad para la malversación de fondos. Asimismo, algunos proyectos de inversión en sectores más sofisticados podrían encubrir futuras ganancias ilegales. De esa forma, el intento de beneficiarse mediante la malversación de fondos redundaría en proyectos de inversión y tecnologías que no necesariamente concordarían con el modelo de producción del país.

Por otra parte, una vez implementadas esas tecnologías y alcanzado un determinado nivel de sofisticación de la estructura de producción del país, la renovación de los proyectos y el reemplazo de máquinas y equipos, entre otras cosas, podrían estimular cada vez más las acciones corruptas y la malversación de fondos. Así, es posible realizar la siguiente interpretación: cuando un país tiene una estructura productiva compleja, con bienes intensivos en tecnología, es más difícil medir esos bienes en comparación con los bienes estándar. Por ejemplo, es más difícil medir la producción de aviones que la de automóviles, porque hay más empresas que producen y venden automóviles que empresas que fabrican aviones. De ese modo, la corrupción puede ocurrir más fácilmente en los países que producen bienes más tecnológicos porque esos sectores pueden concentrarse en un número reducido de empresas (oligopolios) y los precios de los equipos para la producción pueden estar sobreestimados (véanse Shleifer y Vishny, 1993; Hines, 1995; Mauro, 1997b y 1998; y Rose-Ackerman, 1997).

De esta manera, se puede entender que los efectos de la corrupción en el desarrollo pueden potenciarse o mitigarse por medio de la sofisticación de la estructura productiva. Esos efectos se analizarán en el modelo econométrico a partir de la interpretación del signo de la variable de interacción. Se espera que la relación de esa variable con el desarrollo socioeconómico sea ambigua: cuanto más compleja sea la estructura productiva del país, mayores pueden ser las oportunidades de malversación de fondos y soborno, que redundan en mayor corrupción y menor desarrollo. Sin embargo, si no hay una influencia concomitante entre la corrupción, la sofisticación de la estructura productiva y el desarrollo del país, o si esa relación es débil, los efectos de la corrupción no prevalecerán, redundando en una mejora en el desarrollo cuanto más sofisticados sean los bienes producidos, incluso con la existencia de corrupción.

Dicho esto, la variable de interacción del indicador (controlcorrup) con las medidas de la estructura productiva (EP) puede tener una relación negativa con el desarrollo - si la mayor sofisticación de la estructura productiva potencia los efectos de la corrupción, de modo que se modifique la influencia del mayor control de la corrupción y acabe por reducir el desarrollo socioeconómico - o una relación 
positiva, si no hay una influencia concomitante o si la mayor sofisticación de la estructura productiva mitiga los efectos de la corrupción en el desarrollo, de modo que el mayor control de esa corrupción aumente el desarrollo.

A partir de la especificación del modelo empírico y de la exposición de la base de datos, se observó que se trata de una estimación con datos de panel. Las ventajas de su empleo, expuestas en Baltagi (2005), incluyen la capacidad de controlar los efectos heterogéneos que tiene cada país o individuo. Además, proporcionan más información sobre los datos, permiten más variabilidad, eficiencia y libertad y menos colinealidad.

Para la estimación de los datos de panel dinámico, Arellano y Bond (1991) y Arellano y Bover (1995) contribuyen a la utilización del método generalizado de momentos (GMM), que posteriormente avanza con el sistema GMM de Blundell y Bond (1998).

La condición de momento que se vuelve necesaria para que haya ortogonalidad se observa en la ecuación (2).

$$
\begin{array}{lll}
E\left[D E_{i, t-2}\left(\mu_{i t}-\mu_{i, t-1}\right)\right]=0 & \forall & t=3, \ldots, T \\
E\left[X_{i, t-2}^{\prime}\left(\mu_{i t}-\mu_{i, t-1}\right)\right]=0 & \forall & t=3, \ldots, T
\end{array}
$$

Así, como explica Baltagi (2005), en la ecuación de primera diferencia, $D E_{t-2}$ es un instrumento válido por estar fuertemente correlacionado con $\left(D E_{i, t-1}-D E_{i, t-2}\right)$ y no estar correlacionado con $\left(\mu_{i t}-\mu_{i, t-1}\right)$. Después de la estimación, se realizarán pruebas para verificar posibles problemas de autocorrelación y validez de los instrumentos.

\section{Estimaciones y pruebas}

\section{Corrupción, estructura productiva y desarrollo social}

Al relacionar la corrupción y la estructura productiva para verificar sus efectos en el desarrollo social, se estimaron las especificaciones de los cuadros 2 y 3 , en las que se utiliza el porcentaje de la población subalimentada como variable dependiente.

En el cuadro 2 es posible observar los efectos del control de la corrupción (controlcorrup) y de la estructura productiva (eci) en la población subalimentada. Este análisis parcial contribuirá a verificar el comportamiento de esas relaciones sin considerar las variables de control.

En la estimación (1) se utiliza solamente el control de la corrupción en nivel. En las estimaciones (2) y (3) se utilizan el control de la corrupción y (eci) en nivel y en la estimación (3) también se consideran sus efectos rezagados. En la estimación (4) se utiliza solamente la variable (corceci) de interacción entre (controlcorrup) y (eci). En la estimación (5) se controlan los efectos de la desigualdad de ingresos y de los productos básicos no agrícolas.

La estimación (1) indica que el aumento del control de la corrupción reduce la población subalimentada. Este resultado está en línea con la literatura (Blackburn, 2012; Sodré, 2014; Dalberto, 2016), pues un mayor control de la corrupción sugiere menores niveles de actividades corruptas en el país, que a su vez mejoran el desarrollo social. Este resultado se mantiene en la estimación (2) y en el rezago de la estimación (3). En nivel, en la estimación (3), el mayor control de la corrupción aumenta la subalimentación. Esto sugiere que los menores niveles de corrupción aumentarían la subalimentación y podría explicarse conforme la literatura teórica y empírica que plantea argumentos y pruebas acerca de los beneficios de la corrupción para los países en desarrollo (Leff, 1964; Nye, 1967; Huntington, 
2002; Li, Xu y Zou, 2000). A partir de los resultados de la estimación (3), pueden sugerirse indicios de una relación no lineal entre la corrupción y el desarrollo, dado el cambio de dirección de los efectos directos del control de la corrupción.

En las estimaciones (2) en nivel y (3) con rezago, los efectos aislados de la sofisticación de la estructura productiva en la población subalimentada resultaron negativos. Así, se entiende que una estructura de producción más sofisticada en el sentido de conocimiento, representada por (eci), se traducirá en mejoras en el desarrollo social del país (Rodrik, 2004; Schteingart, 2015). Al considerar la variable de interacción (corceci), la estimación (4) muestra que la estructura productiva más sofisticada mitiga los efectos del control de la corrupción en la subalimentación.

\section{Cuadro 2}

Países en desarrollo seleccionados (88): modelo básico de panel dinámico con la subalimentación como variable dependiente, 2002-2014

\begin{tabular}{|c|c|c|c|c|c|}
\hline Variables & (1) & (2) & (3) & (4) & (5) \\
\hline \multirow[t]{2}{*}{$\operatorname{sub}(t-1)$} & $0,902^{\star \star \star}$ & $0,925^{\star \star \star}$ & $0,909^{\star \star \star}$ & $0,948^{\star \star \star}$ & $0,882^{* \star \star}$ \\
\hline & $(0,00986)$ & $(0,0141)$ & $(0,0153)$ & $(0,00970)$ & $(0,0608)$ \\
\hline \multirow[t]{2}{*}{ controlcorrup } & $-0,315^{\star \star}$ & $-0,777^{\star \star \star}$ & $1,324^{\star *}$ & & $-1,789^{\star}$ \\
\hline & $(0,133)$ & $(0,163)$ & $(0,645)$ & & $(0,996)$ \\
\hline \multirow[t]{2}{*}{ controlcorrup(t-1) } & & & $-1,534^{\star \star}$ & & \\
\hline & & & $(0,693)$ & & \\
\hline \multirow[t]{2}{*}{ eci } & & $-0,239^{\star *}$ & $-0,138$ & & \\
\hline & & $(0,0951)$ & $(0,118)$ & & \\
\hline \multirow[t]{2}{*}{ eci(t-1) } & & & $-0,251^{* *}$ & & \\
\hline & & & $(0,128)$ & & \\
\hline \multirow[t]{2}{*}{ corceci } & & & & $0,719^{\star \star \star}$ & \\
\hline & & & & $(0,272)$ & \\
\hline \multirow[t]{2}{*}{ gini } & & & & & $0,123^{\star \star \star}$ \\
\hline & & & & & $(0,0431)$ \\
\hline \multirow[t]{2}{*}{ ores_metal } & & & & & 0,00696 \\
\hline & & & & & $(0,00784)$ \\
\hline \multirow[t]{2}{*}{ Constante } & $1,190^{\star \star \star}$ & $0,919^{\star \star \star}$ & $0,891^{\star \star \star}$ & $0,481^{\star \star \star}$ & $-3,907^{* * *}$ \\
\hline & $(0,181)$ & $(0,193)$ & $(0,201)$ & $(0,141)$ & $(1,441)$ \\
\hline Número de instrumentos & 38 & 72 & 72 & 80 & 24 \\
\hline Prueba de Sargan (Prob>chi2) & 0,4578 & 0,1216 & 0,1849 & 0,1527 & 0,4849 \\
\hline Prueba de Arellano-Bond (autocorrelación "AR(2)”) & 0,9728 & 0,7464 & 0,3945 & 0,5831 & 0,1525 \\
\hline Observaciones & 1056 & 916 & 893 & 916 & 266 \\
\hline Número de países & 88 & 85 & 78 & 85 & 24 \\
\hline
\end{tabular}

Fuente: Elaboración propia.

Nota: Algunas estimaciones corresponden a un número menor de países debido a la no disponibilidad de datos sobre las variables explicativas. Para la variable gini, existen 24 países: Argentina, Armenia, Belarús, Bolivia (Estado Plurinacional de), Brasil, Colombia, Costa Rica, Ecuador, El Salvador, Federación de Rusia, Georgia, Honduras, Kazajstán, México, Panamá, Paraguay, Perú, República de Moldova, República Dominicana, Serbia, Tailandia, Turquía, Ucrania, Uruguay. Para las otras variables, faltan los siguientes países: variable sub, no incluye: Bahamas, Bahrein, Burundi, Hong Kong (RAE de China), Libia, Papua Nueva Guinea, Qatar, República Democrática del Congo, Singapur y Sudán; variables eci y corceci, Bahamas, Brunei Darussalam, Burundi, Gambia, Guinea-Bissau, Guyana, República Democrática del Congo, Sierra Leona, Suriname; variables gdpind, gdpagro, gdpser, corind, coragro y corser, Angola, Bahrein, Kuwait, Liberia, Libia; variable fdii, Cuba; variable cre, Cuba, Irán; variable tech, Angola, Guinea-Bissau, Liberia, Libia, República Democrática del Congo; variable agua, Brunei Darussalam, Hong Kong (RAE de China), Libia; variable hc, Azerbaiyán, Bahamas, Belarús, Cuba, Georgia, Guinea, Guinea-Bissau, Guyana, Líbano, Libia, Omán, Papua Nueva Guinea, Suriname; variable ores_metal, Liberia, República Democrática del Congo. Error estándar entre paréntesis *** $p<0,01,{ }^{* *} p<0,05,{ }^{*} p<0,1$. 


\section{Cuadro 3}

Países en desarrollo seleccionados (88): panel dinámico con la subalimentación como variable dependiente, 2002-2014

\begin{tabular}{|c|c|c|c|c|c|c|}
\hline Variables & (1) & (2) & (3) & (4) & (5) & (6) \\
\hline \multirow{2}{*}{$\operatorname{sub}_{(t-1)}$} & $0,864^{\star \star \star}$ & $0,811^{\text {***}}$ & $0,880^{\star \star \star}$ & $0,827^{\star \star \star}$ & $0,788^{\star \star \star}$ & $0,745^{\star \star \star}$ \\
\hline & $(0,0305)$ & $(0,0294)$ & $(0,0273)$ & $(0,0165)$ & $(0,0244)$ & $(0,0824)$ \\
\hline \multirow[t]{2}{*}{ controlcorrup } & $1,127^{\star \star \star}$ & $-8,069^{\star \star \star}$ & $-3,370^{*}$ & $1,891^{\star \star \star}$ & $10,39^{\star \star}$ & $-3,387^{\star \star \star}$ \\
\hline & $(0,367)$ & $(2,577)$ & $(2,027)$ & $(0,707)$ & $(4,846)$ & $(1,128)$ \\
\hline \multirow[t]{2}{*}{ corceci } & $-0,0703$ & & & & & $-1,756$ \\
\hline & $(0,865)$ & & & & & $(3,537)$ \\
\hline \multirow[t]{2}{*}{$\operatorname{corceci}_{(t-1)}$} & $2,440^{\star \star \star}$ & & & & & $1,457^{\star \star}$ \\
\hline & $(0,797)$ & & & & & $(0,696)$ \\
\hline \multirow[t]{2}{*}{ corind } & & $0,314^{\star \star \star}$ & $0,170^{\star *}$ & & & \\
\hline & & $(0,0859)$ & $(0,0708)$ & & & \\
\hline \multirow[t]{2}{*}{ corind $_{(t-1)}$} & & $-0,0652^{\star \star}$ & $-0,0644^{\star \star}$ & & & \\
\hline & & $(0,0270)$ & $(0,0268)$ & & & \\
\hline \multirow[t]{2}{*}{ coragro } & & & & $-0,0514$ & & \\
\hline & & & & $(0,0476)$ & & \\
\hline \multirow[t]{2}{*}{ coragro $_{(t-1)}$} & & & & $-0,0686^{\star \star}$ & & \\
\hline & & & & $(0,0288)$ & & \\
\hline \multirow[t]{2}{*}{ corser } & & & & & $-0,158^{\star}$ & \\
\hline & & & & & $(0,0919)$ & \\
\hline \multirow{2}{*}{$\operatorname{corser}_{(t-1)}$} & & & & & $-0,0344^{\star *}$ & \\
\hline & & & & & $(0,0141)$ & \\
\hline \multirow[t]{2}{*}{ eci } & 0,0698 & & & & & 0,750 \\
\hline & $(0,325)$ & & & & & $(1,091)$ \\
\hline \multirow{2}{*}{$e c i_{(t-1)}$} & $-0,633^{* *}$ & & & & & \\
\hline & $(0,314)$ & & & & & \\
\hline \multirow[t]{2}{*}{ gdpind } & & $-0,149^{\star \star \star}$ & $-0,0916^{\star \star \star}$ & $-0,0165$ & $-0,0159$ & \\
\hline & & $(0,0432)$ & $(0,0299)$ & $(0,0140)$ & $(0,0202)$ & \\
\hline \multirow{2}{*}{$\operatorname{gdpind}_{(t-1)}$} & & $0,0616^{\star \star \star}$ & $0,0539^{\star \star \star}$ & $0,0319^{\star \star}$ & $0,0312^{\star \star}$ & \\
\hline & & $(0,0176)$ & $(0,0187)$ & $(0,0129)$ & $(0,0125)$ & \\
\hline \multirow[t]{2}{*}{ gdpagro } & & & $0,0335^{\star}$ & $0,0390^{\star \star}$ & & \\
\hline & & & $(0,0202)$ & $(0,0190)$ & & \\
\hline \multirow[t]{2}{*}{ gdpser } & & 0,00729 & & & $0,0605^{\star}$ & \\
\hline & & $(0,0267)$ & & & $(0,0316)$ & \\
\hline \multirow[t]{2}{*}{ fdii } & $-0,0166$ & $0,0400^{* *}$ & $-0,00182$ & 0,00127 & $0,0297^{* *}$ & $-0,0875^{\star}$ \\
\hline & $(0,0132)$ & $(0,0165)$ & $(0,0133)$ & $(0,0117)$ & $(0,0140)$ & $(0,0509)$ \\
\hline \multirow[t]{2}{*}{$h c$} & $-0,0977^{*}$ & $-1,246^{\star \star}$ & $-1,508^{\star \star \star}$ & $-1,710^{\star \star \star}$ & $-2,069^{\star \star \star}$ & $-1,651^{\star \star *}$ \\
\hline & $(0,0565)$ & $(0,519)$ & $(0,313)$ & $(0,153)$ & $(0,349)$ & $(0,571)$ \\
\hline \multirow[t]{2}{*}{ agua } & $-0,0738^{\star \star \star}$ & $-0,0303$ & $0,0503^{\star *}$ & & & $-0,115^{\star}$ \\
\hline & $(0,0196)$ & $(0,0309)$ & $(0,0254)$ & & & $(0,0634)$ \\
\hline \multirow[t]{2}{*}{ gini } & & & & & & 0,0207 \\
\hline & & & & & & $(0,0233)$ \\
\hline \multirow[t]{2}{*}{ ores_metal } & & & & & & $0,0982^{\star \star}$ \\
\hline & & & & & & $(0,0448)$ \\
\hline Constante & $7,897^{\star \star \star}$ & $10,16^{\star \star \star}$ & 1,343 & $4,977^{\star \star \star}$ & $3,695^{\star \star}$ & $17,24^{\star \star}$ \\
\hline & $(2,087)$ & $(2,861)$ & $(2,397)$ & $(0,792)$ & $(1,696)$ & $(6,846)$ \\
\hline Número de instrumentos & 74 & 80 & 80 & 80 & 80 & 30 \\
\hline Prueba de Sargan (Prob>chi2) & 0,9406 & 0,6768 & 0,8676 & 0,6925 & 0,1275 & 0,9563 \\
\hline $\begin{array}{l}\text { Prueba de Arellano-Bond } \\
\text { (autocorrelación "AR(2)") }\end{array}$ & 0,8147 & 0,2314 & 0,7585 & 0,6243 & 0,2828 & 0,4327 \\
\hline Observaciones & 811 & 863 & 865 & 878 & 876 & 212 \\
\hline Número de países & 71 & 74 & 74 & 75 & 75 & 21 \\
\hline
\end{tabular}

Fuente: Elaboración propia.

Nota: Algunas especificaciones son similares, pues se intentó verificar la robustez de los resultados de las variables de interacción. En el caso de algunas variables no se dispone de información sobre todos los países. Para la variable gini, existen 24 países: Argentina, Armenia, Belarús, Bolivia (Estado Plurinacional de), Brasil, Colombia, Costa Rica, Ecuador, El Salvador, Federación de Rusia, Georgia, Honduras, Kazajstán, México, Panamá, Paraguay, Perú, República de Moldova, República Dominicana, Serbia, Tailandia, Turquía, Ucrania, Uruguay. Para las otras variables, faltan los siguientes países: variable sub, no incluye: Bahamas, Bahrein, Burundi, Hong Kong (RAE de China), Libia, Papua Nueva Guinea, Qatar, República Democrática del Congo, Singapur y Sudán; variables eci y corceci, Bahamas, Brunei Darussalam, Burundi, Gambia, Guinea-Bissau, Guyana, República Democrática del Congo, Sierra Leona, Suriname; variables gdpind, gdpagro, gdpser, corind, coragro y corser, Angola, Bahrein, Kuwait, Liberia, Libia; variable fdii, Cuba; variable cre, Cuba, Irán; variable tech, Angola, Guinea-Bissau, Liberia, Libia, República Democrática del Congo; variable agua, Brunei Darussalam, Hong Kong (RAE de China), Libia; variable hc, Azerbaiyán, Bahamas, Belarús, Cuba, Georgia, Guinea, Guinea-Bissau, Guyana, Líbano, Libia, Omán, Papua Nueva Guinea, Suriname; variable ores_metal, Liberia, República Democrática del Congo.

Error estándar entre paréntesis ${ }^{\star \star \star} p<0,01,{ }^{* \star} p<0,05,{ }^{*} p<0,1$. 
Podría argumentarse que la sofisticación de la estructura productiva contribuiría a los efectos de la corrupción como "lubricante de las ruedas", en el sentido de que un menor control de las acciones corruptas reduciría la proporción de la población subalimentada. Eso también se puede interpretar de la siguiente manera: independientemente de que el efecto de la propia corrupción sea perverso o no sobre la subalimentación, la estructura productiva más sofisticada potencia ese efecto de la corrupción, así que, en ese contexto, aumentar el control de la corrupción no reduce la desnutrición.

En la estimación de control (5), las variables generalmente mantienen el mismo signo de las demás estimaciones. El sector de los productos básicos no agrícolas fue insignificante y la desigualdad de ingresos contribuyó al aumento de la subalimentación.

En el cuadro 3 se presentan las estimaciones que incluyen variables de control. En la estimación (1) se utilizan el control de la corrupción (controlcorrup), la sofisticación de la producción (eci) y la variable de interacción (corceci), controlando la inversión extranjera directa, el capital humano y la proporción de la población que utiliza fuentes de agua potable mejoradas. La diferencia entre las estimaciones (2) y (3) es que en la estimación (2) se considera también el PIB de servicios, mientras en la estimación (3) se considera el PIB agrícola. En la estimación (4) se utilizan el control de la corrupción, el PIB agrícola, la variable de interacción con el PIB agrícola, el PIB de la industria, la inversión extranjera directa y el capital humano. En la estimación (5) se consideran el control de la corrupción, el PIB de servicios, la variable de interacción con el PIB de servicios, el PIB industrial, la inversión extranjera directa y el capital humano. En la estimación (6) se controlan los efectos de la desigualdad de ingresos y de los productos básicos no agrícolas.

En el caso de las estimaciones que controlan otros efectos, cabe analizar también el rezago de la variable dependiente. Los coeficientes de esas variables tuvieron un valor de alrededor de 0,80, que puede representar el efecto dinámico de la subalimentación a lo largo del tiempo. Por lo tanto, la subalimentación estuvo influenciada de manera persistente por el nivel de subalimentación que el país registró en períodos anteriores. Esto indica la dificultad y el posible retraso en la transición de los países menos desarrollados. Dada la persistencia del pasado, este resultado sugiere que las políticas implementadas tendrán poco impacto inmediato en la subnutrición.

Los efectos del control de la corrupción fueron sensibles a las variables de control utilizadas: en las estimaciones (2) y (3), en las que se utilizó la interacción del control de la corrupción con el PIB del sector industrial, el control de la corrupción tuvo un impacto negativo directo en la subalimentación. En las demás estimaciones, el coeficiente presentado resultó positivo. Esta sensibilidad de los efectos del control de la corrupción en el desarrollo social refuerza los indicios de una relación no lineal.

Con respecto a las variables de interacción, al considerar el rezago de la interacción del control de la corrupción tanto con el PIB industrial (corind) en las estimaciones (2) y (3), como con la agricultura (coragro) en la estimación (4) y los servicios (corser) en la estimación (5), hubo un impacto negativo en la subalimentación. Los resultados muestran, por lo tanto, que este caso - en que el rezago de las estructuras productivas más o menos sofisticadas que interactúan con el control de la corrupción afecta del mismo modo el desarrollo social - remite a la idea de que posiblemente la estructura del país no mitigará ni potenciará los efectos pasados de la corrupción. En contrapartida, el resultado de la variable rezagada (corceci) muestra nuevamente que el control de la corrupción aumenta la subalimentación.

Al considerar las interacciones en nivel se observa que, en el caso de la estructura productiva relativamente menos sofisticada representada por el PIB de servicios en la estimación (5), se potenciaron los efectos negativos esperados del control de la corrupción en la subalimentación (interacción negativa). En otras palabras, cuanto mayor sea el control de la corrupción en una estructura menos sofisticada, menor será la subalimentación. En el caso de la estructura más sofisticada representada por la industria en las estimaciones (2) y (3), los efectos negativos esperados del control de la corrupción en la subalimentación se mitigaron, generando una interacción positiva. 
Una vez más, esta idea puede interpretarse de la siguiente manera: al esperarse que un mayor control de la corrupción disminuya la subalimentación y resultar positiva la variable de interacción, se sugiere que la estructura productiva más sofisticada mitiga o invierte los efectos del mayor control de la corrupción en la subalimentación, o más aún, que esa estructura potencia los efectos de la corrupción en la subalimentación, en cuyo caso se puede especular que la corrupción sería beneficiosa para reducir la subalimentación. Se sugiere entonces que una estructura productiva más sofisticada distorsiona los efectos esperados del control de la corrupción en el desarrollo, de modo que prevalecen los efectos de la corrupción y no los de su control (Shleifer y Vishny, 1993; Rose-Ackerman, 1997; Mauro, 1997b, 1998), mientras que, en el marco de una estructura menos sofisticada, prevalecen los efectos del control de dicha corrupción.

En el caso de las demás variables de interés, hubo indicios de que un aumento del PIB agrícola (gdpagro) no necesariamente reduce la subalimentación (véanse Rao y Caballero, 1990; Reis, 2012), mientras el PIB industrial (gdpind) en nivel tuvo efectos negativos en la subalimentación, pero su valor rezagado indicó un aumento de esta. Esta última relación es coherente con la literatura, pues la parte industrial no necesariamente tendrá relaciones directas con las variables sociales de la economía. La parte de servicios del PIB (gdpser) resultó en un aumento de la subalimentación. Por último, la complejidad económica (eci) en su valor rezagado disminuyó la subalimentación, conforme lo esperado (Hartmann y otros, 2015).

Con respecto a las variables de control se observa que, en el caso del conjunto de países en desarrollo seleccionados, si bien la inversión extranjera directa y la calidad del agua pudieron contribuir a reducir la subalimentación, este resultado es sensible a la especificación. Por otra parte, el capital humano contribuyó a reducir la subalimentación en todas las estimaciones.

En la estimación de control (6), las variables generalmente mantienen el mismo signo de las demás estimaciones. La desigualdad de ingresos fue insignificante, y el sector de productos básicos no agrícolas contribuyó al aumento de la subalimentación, pues los recursos no agrícolas posiblemente no tendrán relaciones directas con las variables sociales, a diferencia de lo que ocurriría, por ejemplo, si se consideraran solamente los agrícolas.

En los cuadros 2 y 3, las pruebas de validez de los instrumentos (Sargan) y las pruebas de autocorrelación (Arellano-Bond) indicaron que los instrumentos fueron válidos en todas las estimaciones y que no hubo indicios de autocorrelación?.

\section{Corrupción, estructura productiva y desarrollo económico}

La relación entre la corrupción y la estructura productiva y el desarrollo económico puede observarse a partir de los cuadros 4 y 5 , en los que se utiliza el logaritmo del PIB per cápita como variable dependiente.

En el cuadro 4 es posible observar los efectos del control de la corrupción y de la estructura productiva en el producto per cápita. En la estimación (1) se utiliza solamente el control de la corrupción en nivel. En las estimaciones (2) y (3) se utilizan el control de la corrupción y (eci) en nivel y en la estimación (3) también se consideran sus efectos rezagados. En la estimación (4) se utiliza solamente la variable de interacción (corceci). En la estimación (5) se controlan los efectos de la desigualdad de ingresos y de los productos básicos no agrícolas.

\footnotetext{
7 Las demás estimaciones de control se encuentran en el anexo A2. Se realizaron pruebas adicionales por regiones, que pueden solicitarse a los autores.
} 


\section{Cuadro 4}

Países en desarrollo seleccionados (98): modelo básico de panel dinámico con el logaritmo del PIB per cápita como variable dependiente, 2002-2014

\begin{tabular}{|c|c|c|c|c|c|}
\hline Variables & (1) & (2) & (3) & (4) & (5) \\
\hline \multirow[t]{2}{*}{$\log d p_{(t-1)}$} & $0,894^{\star \star \star}$ & $1,051^{\text {***}}$ & $0,822^{\star \star \star}$ & $0,981^{\star \star \star}$ & $0,895^{\star \star \star}$ \\
\hline & $(0,0196)$ & $(0,0246)$ & $(0,0664)$ & $(0,0101)$ & $(0,0332)$ \\
\hline \multirow[t]{2}{*}{ controlcorrup } & $0,350^{\text {*** }}$ & $-0,106^{\star *}$ & $-0,287$ & & $0,268^{\star \star *}$ \\
\hline & $(0,0702)$ & $(0,0470)$ & $(0,184)$ & & $(0,0996)$ \\
\hline \multirow{2}{*}{$\operatorname{controlcorrup}_{(t-1)}$} & & & $0,445^{\star \star}$ & & \\
\hline & & & $(0,174)$ & & \\
\hline \multirow[t]{2}{*}{ eci } & & $-0,0304$ & 0,0486 & & \\
\hline & & $(0,0188)$ & $(0,0406)$ & & \\
\hline \multirow{2}{*}{$e c i_{(t-1)}$} & & & $0,171^{\star \star}$ & & \\
\hline & & & $(0,0683)$ & & \\
\hline \multirow[t]{2}{*}{ corceci } & & & & $0,0643^{\star \star \star}$ & \\
\hline & & & & $(0,0246)$ & \\
\hline \multirow[t]{2}{*}{ gini } & & & & & 0,000841 \\
\hline & & & & & $(0,000635)$ \\
\hline \multirow[t]{2}{*}{ ores_metal } & & & & & $-0,000538^{*}$ \\
\hline & & & & & $(0,000309)$ \\
\hline \multirow[t]{2}{*}{ Constante } & $0,749^{\star \star \star}$ & $-0,359^{*}$ & $1,505^{\star \star \star}$ & $0,192^{\star \star}$ & $0,801^{\star \star \star}$ \\
\hline & $(0,133)$ & $(0,192)$ & $(0,531)$ & $(0,0850)$ & $(0,236)$ \\
\hline Número de instrumentos & 92 & 56 & 34 & 85 & 26 \\
\hline Prueba de Sargan (Prob>chi2) & 0,1039 & 0,2427 & 0,5369 & 0,1597 & 0,9875 \\
\hline Prueba de Arellano-Bond (autocorrelación “AR(2)”) & 0,4665 & 0,4303 & 0,6330 & 0,4292 & 0,0110 \\
\hline Observaciones & 1174 & 934 & 966 & 905 & 266 \\
\hline Número de países & 98 & 83 & 85 & 82 & 24 \\
\hline
\end{tabular}

Fuente: Elaboración propia.

Nota: Algunas estimaciones corresponden a un número menor de países debido a la no disponibilidad de datos sobre las variables explicativas. Para la variable gini, existen 24 países: Argentina, Armenia, Belarús, Bolivia (Estado Plurinacional de), Brasil, Colombia, Costa Rica, Ecuador, El Salvador, Federación de Rusia, Georgia, Honduras, Kazajstán, México, Panamá, Paraguay, Perú, República de Moldova, República Dominicana, Serbia, Tailandia, Turquía, Ucrania, Uruguay. Para las otras variables, faltan los siguientes países: variable sub, no incluye: Bahamas, Bahrein, Burundi, Hong Kong (RAE de China), Libia, Papua Nueva Guinea, Qatar, República Democrática del Congo, Singapur y Sudán; variables eci y corceci, Bahamas, Brunei Darussalam, Burundi, Gambia, Guinea-Bissau, Guyana, República Democrática del Congo, Sierra Leona, Suriname; variables gdpind, gdpagro, gdpser, corind, coragro y corser, Angola, Bahrein, Kuwait, Liberia, Libia; variable fdii, Cuba; variable cre, Cuba, Irán; variable tech, Angola, Guinea-Bissau, Liberia, Libia, República Democrática del Congo; variable agua, Brunei Darussalam, Hong Kong (RAE de China), Libia; variable hc, Azerbaiyán, Bahamas, Belarús, Cuba, Georgia, Guinea, Guinea-Bissau, Guyana, Líbano, Libia, Omán, Papua Nueva Guinea, Suriname; variable ores_metal, Liberia, República Democrática del Congo. Error estándar entre paréntesis ${ }^{* \star} p<0,01,{ }^{* \star} p<0,05,{ }^{*} p<0,1$.

En las estimaciones (1) y (3) hay indicios de que un aumento del control de la corrupción incrementa el producto per cápita, en consonancia con la literatura (Mauro, 1995, 1997a; Akçay, 2006). Sin embargo, en la estimación (2) el coeficiente negativo está en línea con la literatura teórica y empírica que plantea los beneficios de la corrupción para los países en desarrollo (Klitgaard, 1994; Acemoglu y Verdier, 1998). Una vez más, el cambio de dirección de los efectos directos del control de la corrupción en el desarrollo sugiere una relación no lineal. El valor rezagado de (eci) fue positivo.

Al considerar la variable de interacción (corceci), la estimación (4) muestra que una estructura productiva más sofisticada potencia los efectos del control de la corrupción. Esto puede indicar la posibilidad de que los efectos de la corrupción en el producto per cápita sean perjudiciales, de ahí la importancia de un mayor control de la corrupción en el marco de una estructura productiva más sofisticada. Por lo tanto, mientras los efectos del control de la corrupción (que se espera sean positivos en el producto) se potencian, los efectos de la corrupción en sí (que se espera sean negativos en el producto) pueden mitigarse. La mitigación de la corrupción en el marco de una estructura productiva 
más sofisticada puede indicar que en realidad no existe una relación entre la sofisticación de la estructura de producción y los actos corruptos.

En la estimación de control (5), las variables generalmente mantienen el mismo signo de las demás estimaciones. El coeficiente de Gini fue insignificante y los productos básicos no agrícolas tuvieron un impacto negativo en el PIB per cápita, a diferencia de lo esperado pero en línea con los resultados de Sachs y Warner (2001).

En el cuadro 5 se consideran algunas variables de control adicionales. En la estimación (1) se utilizan el control de la corrupción (controlcorrup), la sofisticación de la producción (eci), la variable de interacción (corceci), la inversión extranjera directa, el capital humano y la proporción de la población que utiliza fuentes de agua potable mejoradas. La diferencia entre las estimaciones (2) y (3) es que en la estimación (2) se considera incluso el PIB agrícola. En la estimación (4) se utilizan el control de la corrupción, el PIB agrícola, el PIB de la industria, la variable de interacción con el PIB agrícola, la inversión extranjera directa, el capital humano y la apertura comercial. La diferencia entre las estimaciones (5) y (6) es que en la estimación (5) también se considera el PIB industrial. En la estimación (7) se controlan los efectos de la desigualdad de ingresos y de los productos básicos no agrícolas.

Los coeficientes de la variable dependiente rezagada fueron positivos y representan el efecto dinámico del PIB per cápita. Sus valores fueron de alrededor de 0,85. La persistencia de los efectos pasados en el PIB per cápita actual sugiere dificultad para que los niveles de PIB cambien de manera considerable y las economías alcancen rápidamente un mayor nivel de desarrollo. En consecuencia, se necesitan políticas económicas eficientes para mejorar esta situación a lo largo del tiempo.

Los efectos del control de la corrupción, tanto en nivel como rezagado, resultaron nuevamente muy sensibles a las variables de control utilizadas. Una vez más, esa sensibilidad puede dar indicios de una relación no lineal entre la corrupción y el desarrollo.

Con respecto a las variables de interacción, se observa que los valores en nivel de la interacción entre la corrupción y el PIB agrícola y de servicios en las estimaciones (4), (5) y (6), que representan una estructura productiva relativamente menos sofisticada, cambian de signo en comparación con sus valores rezagados de las estimaciones (4) y (6). En nivel, los efectos positivos esperados del control de la corrupción en el PIB per cápita se potencian en una estructura productiva menos sofisticada. En otras palabras, cuando la sofisticación productiva es menor, resulta más fácil controlar la corrupción, de manera que los efectos de la corrupción (posiblemente negativos en el producto) se mitigan y esto produce un aumento en el PIB per cápita. En este caso, se puede sugerir que la corrupción tendría efectos negativos en el desarrollo económico, pues su control genera una mejora en el producto per cápita. Además, se sugiere que, en el marco de una estructura productiva menos sofisticada, los efectos del control de la corrupción prevalecen sobre los efectos de la corrupción misma. 


\section{Cuadro 5}

Países en desarrollo seleccionados (98): panel dinámico con el logaritmo del PIB per cápita como variable dependiente, 2002-2014

\begin{tabular}{|c|c|c|c|c|c|c|c|}
\hline Variables & (1) & (2) & (3) & (4) & (5) & (6) & (7) \\
\hline \multirow{2}{*}{$\log g d p_{(t-1)}$} & $0,748^{\star \star \star}$ & $0,892^{\star \star \star}$ & $0,861^{\star * \star}$ & $0,921^{\star \star \star}$ & $0,826^{\star \star \star}$ & $0,859^{\star \star \star}$ & $0,920^{\star \star \star}$ \\
\hline & $(0,0544)$ & $(0,0364)$ & $(0,0575)$ & $(0,0325)$ & $(0,0605)$ & $(0,0636)$ & $(0,0710)$ \\
\hline \multirow[t]{2}{*}{ controlcorrup } & $0,235^{\star \star \star}$ & 0,206 & 0,301 & $-0,205$ & $-0,692^{\star \star}$ & $-1,177^{*}$ & 2,099 \\
\hline & $(0,0644)$ & $(0,205)$ & $(0,259)$ & $(0,127)$ & $(0,340)$ & $(0,603)$ & $(1,374)$ \\
\hline \multirow{2}{*}{ controlcorrup $_{(t-1)}$} & & $-0,422^{\star \star}$ & $-0,702^{\star \star}$ & $0,274^{\star \star}$ & & $0,960^{*}$ & $1,922^{*}$ \\
\hline & & $(0,211)$ & $(0,282)$ & $(0,134)$ & & $(0,554)$ & $(1,158)$ \\
\hline \multirow[t]{2}{*}{ corceci } & $-0,0921$ & & & & & & \\
\hline & $(0,0883)$ & & & & & & \\
\hline \multirow[t]{2}{*}{ corind } & & $-0,00847$ & $-0,00678$ & & & & $-0,0705^{\star}$ \\
\hline & & $(0,00720)$ & $(0,00887)$ & & & & $(0,0428)$ \\
\hline \multirow{2}{*}{ corind $_{(t-1)}$} & & $0,0199^{\star \star \star}$ & $0,0291^{\star \star \star}$ & & & & $-0,0518$ \\
\hline & & $(0,00764)$ & $(0,0102)$ & & & & $(0,0340)$ \\
\hline \multirow[t]{2}{*}{ coragro } & & & & $0,0103^{*}$ & & & \\
\hline & & & & $(0,00564)$ & & & \\
\hline \multirow[t]{2}{*}{ coragro $_{(t-1)}$} & & & & $-0,00896^{*}$ & & & \\
\hline & & & & $(0,00488)$ & & & \\
\hline \multirow[t]{2}{*}{ corser } & & & & & $0,0174^{\star \star \star}$ & $0,0219^{\star *}$ & \\
\hline & & & & & $(0,00626)$ & $(0,0109)$ & \\
\hline \multirow{2}{*}{$\operatorname{corser}_{(t-1)}$} & & & & & & $-0,0179^{*}$ & \\
\hline & & & & & & $(0,0105)$ & \\
\hline \multirow[t]{2}{*}{ eci } & 0,0376 & & & & & & \\
\hline & $(0,0342)$ & & & & & & \\
\hline \multirow[t]{2}{*}{ gdpind } & & $0,0151^{\star \star \star}$ & $0,0154^{\star \star \star}$ & $0,0120^{\star \star \star}$ & $-0,00353$ & & $0,049^{\star \star \star}$ \\
\hline & & $(0,00294)$ & $(0,00348)$ & $(0,00124)$ & $(0,00323)$ & & $(0,0163)$ \\
\hline \multirow[t]{2}{*}{ gdpind $_{(t-1)}$} & & $-0,0179^{\star \star \star}$ & $-0,0195^{\star \star \star}$ & $-0,0105^{\star \star \star}$ & & & $-0,00684$ \\
\hline & & $(0,00318)$ & $(0,00428)$ & $(0,00137)$ & & & $(0,0127)$ \\
\hline \multirow{2}{*}{ gdpagro } & & $-0,000506$ & & $-0,00395^{\star}$ & & & $0,019^{\star \star \star}$ \\
\hline & & $(0,00241)$ & & $(0,00222)$ & & & $(0,0075)$ \\
\hline \multirow{2}{*}{ gdpagro $_{(t-1)}$} & & $-0,00519^{\star \star}$ & & & & & $-0,03^{\star \star \star}$ \\
\hline & & $(0,00211)$ & & & & & $(0,0069)$ \\
\hline \multirow[t]{2}{*}{ gdpser } & & & & & $-0,0184^{\star \star \star}$ & $-0,0194^{\star * \star}$ & \\
\hline & & & & & $(0,00418)$ & $(0,00430)$ & \\
\hline \multirow{2}{*}{$\operatorname{gdpser}_{(t-1)}$} & & & & & $0,00644^{\star \star *}$ & $0,0172^{\star \star \star}$ & \\
\hline & & & & & $(0,00135)$ & $(0,00446)$ & \\
\hline \multirow[t]{2}{*}{ fdii } & $0,00628^{\star \star \star}$ & & & 0,000544 & 0,00194 & $-0,000268$ & \\
\hline & $(0,00159)$ & & & $(0,000334)$ & $(0,00138)$ & $(0,00216)$ & \\
\hline \multirow[t]{2}{*}{$h c$} & $0,149^{\star \star \star}$ & $0,0822^{\star \star \star}$ & $0,187^{\star \star}$ & $0,0642^{\star \star \star}$ & $0,331^{\star \star \star}$ & $0,241^{\star \star}$ & $0,265^{*}$ \\
\hline & $(0,0339)$ & $(0,0232)$ & $(0,0765)$ & $(0,0201)$ & $(0,0811)$ & $(0,104)$ & $(0,136)$ \\
\hline agua & $\begin{array}{l}0,0101^{\star \star \star} \\
(0,00222)\end{array}$ & & & & & & \\
\hline abecom & & & & 0,000135 & & & \\
\hline & & & & $(9,13 e-05)$ & & & \\
\hline gini & & & & & & & 0,00451 \\
\hline & & & & & & & $(0,0049)$ \\
\hline ores_metal & & & & & & & $-0,00540$ \\
\hline & & & & & & & $(0,0044)$ \\
\hline Constante & $\frac{0,764^{\star \star \star}}{(0,159)}$ & $\begin{array}{l}0,824^{\star \star \star} \\
(0,284)\end{array}$ & $\frac{0,730^{\star \star \star}}{(0,255)}$ & $\frac{0,484^{* \star}}{(0,199)}$ & $\frac{1,303^{\star \star \star}}{(0,265)}$ & $\begin{array}{c}0,724^{*} \\
(0,374)\end{array}$ & $\frac{-1,512^{\star \star}}{(0,714)}$ \\
\hline Número de instrumentos & 77 & 81 & 52 & 84 & 65 & 45 & 27 \\
\hline Prueba de Sargan (Prob>chi2) & 0,1976 & 0,7973 & 0,3435 & 0,5232 & 0,2300 & 0,8234 & 0,2546 \\
\hline $\begin{array}{l}\text { Prueba de Arellano-Bond } \\
\text { (autocorrelación "AR(2)") }\end{array}$ & 0,1752 & 0,1038 & 0,0917 & 0,936 & 0,1181 & 0,2665 & 0,9824 \\
\hline Observaciones & 873 & 950 & 950 & 937 & 947 & 947 & 237 \\
\hline Número de países & 83 & 81 & 81 & 81 & 81 & 81 & 22 \\
\hline
\end{tabular}

Fuente: Elaboración propia.

Nota: Algunas especificaciones son similares, pues se intentó verificar la robustez de los resultados de las variables de interacción. En el caso de algunas variables no se dispone de información sobre todos los países. Para la variable gini, existen 24 países: Argentina, Armenia, Belarús, Bolivia (Estado Plurinacional de), Brasil, Colombia, Costa Rica, Ecuador, El Salvador, Federación de Rusia, Georgia, Honduras, Kazajstán, México, Panamá, Paraguay, Perú, República de Moldova, República Dominicana, Serbia, Tailandia, Turquía, Ucrania, Uruguay. Para las otras variables, faltan los siguientes países: variable sub, no incluye: Bahamas, Bahrein, Burundi, Hong Kong (RAE de China), Libia, Papua Nueva Guinea, Qatar, República Democrática del Congo, Singapur y Sudán; variables eci y corceci, Bahamas, Brunei Darussalam, Burundi, Gambia, Guinea-Bissau, Guyana, República Democrática del Congo, Sierra Leona, Suriname; variables gdpind, gdpagro, gdpser, corind, coragro y corser, Angola, Bahrein, Kuwait, Liberia, Libia; variable fdii, Cuba; variable cre, Cuba, Irán; variable tech, Angola, Guinea-Bissau, Liberia, Libia, República Democrática del Congo; variable agua, Brunei Darussalam, Hong Kong (RAE de China), Libia; variable hc, Azerbaiyán, Bahamas, Belarús, Cuba, Georgia, Guinea, Guinea-Bissau, Guyana, Líbano, Libia, Omán, Papua Nueva Guinea, Suriname; variable ores_metal, Liberia, República Democrática del Congo.

Error estándar entre paréntesis ${ }^{\star \star \star} p<0,01,{ }^{* \star} p<0,05,{ }^{*} p<0,1$. 
Con los valores rezagados, los efectos de las variables de interacción se modificaron. Esto indica que, incluso en el rezago, cuando se trata de desarrollo económico, la corrupción puede tener diferentes efectos según la sofisticación de la estructura productiva del país. Al considerar la interacción en valor rezagado con el PIB agrícola y el PIB de servicios, que representan bajos niveles de sofisticación de la estructura productiva con respecto al sector industrial, los efectos del control de la corrupción (que se espera mejoren los valores del PIB per cápita) en realidad se mitigan o invierten debido a la baja sofisticación productiva. Por el contrario, al considerar la interacción con el PIB industrial en las estimaciones (2) y (3), los efectos del control de la corrupción se potencian debido a la mayor sofisticación productiva. En otras palabras, a pesar de que las diferentes estructuras productivas rezagadas afectan de diferente manera el PIB per cápita, esos efectos no coinciden con la interpretación de que una estructura productiva más sofisticada potencia los efectos de la corrupción al distorsionar los efectos del control de la corrupción en los actos corruptos, como sugieren los resultados de los cuadros 2 y 3 y los valores en nivel del cuadro 5. Así, el argumento de "lubricación de las ruedas" por la corrupción, en este caso de las variables rezagadas para el desarrollo económico, estaría asociado con una estructura productiva menos sofisticada.

En el caso de las demás variables de interés, el PIB agrícola afectó negativamente el PIB per cápita en nivel y en el rezago, como se sugiere en algunos estudios de la literatura (Sachs y Warner, 2001), pues puede entenderse que el efecto de la agricultura es mayor en términos sociales que en términos económicos, que estarían más fuertemente influenciados por la industria. Si bien el PIB industrial en nivel aumentó el PIB per cápita, su valor en el rezago tuvo un impacto negativo. Por otra parte, mientras el PIB de servicios en nivel disminuyó el PIB per cápita, posiblemente por la misma razón que el sector agrícola, sus valores rezagados mejoraron el PIB per cápita actual.

Con respecto a las variables de control, se observa que la inversión extranjera directa y las fuentes de agua potable mejoradas aumentaron el PIB per cápita en la estimación (1) y que el capital humano tuvo el mismo efecto en todas las estimaciones.

En la estimación de control (7), las variables generalmente presentan el mismo signo que las demás estimaciones. La variable de interacción (corind) en nivel tuvo un impacto negativo en el PIB per cápita, corroborando la hipótesis de distorsión de los efectos esperados del control de la corrupción en el marco de una estructura productiva relativamente más sofisticada. El coeficiente de Gini y los productos básicos no agrícolas fueron insignificantes.

En los cuadros 4 y 5, las pruebas de validez de los instrumentos (Sargan) indicaron que los instrumentos fueron válidos en todas las estimaciones ${ }^{8}$. Las pruebas de autocorrelación (ArellanoBond) indicaron ausencia de autocorrelación, con excepción de las estimaciones (5) del cuadro 4 y (3) del cuadro 5.

\section{Análisis no lineal}

Si bien el sentido común indica que la corrupción debería afectar negativamente las economías de los países, como en Mauro (1997a), sus efectos son controvertidos, no solamente en el campo teórico sino también en los estudios empíricos. Algunos autores que argumentan sobre los beneficios de la corrupción son: Leff (1964), Nye (1967), Huntington (2002), Klitgaard (1994), Bardhan (1997) y Acemoglu y Verdier (1998).

Con respecto a la corrupción, la estructura productiva y sus efectos en el desarrollo de los países, se puede considerar que la causalidad sugerida es bidireccional. Explorar este aspecto puede ayudar a profundizar el análisis. Los efectos del control de la corrupción se invertirían, pues la existencia de

\footnotetext{
8 Las demás estimaciones de control se encuentran en el anexo A3. Se pueden solicitar pruebas adicionales a los autores.
} 
corrupción podría, por ejemplo, afectar positivamente el PIB per cápita y negativamente la variable de subalimentación al estimular inversiones en infraestructura y capital físico. Así, de haber corrupción, las personas estarían dispuestas a optar por tecnologías más avanzadas o proyectos de inversión en sectores más sofisticados con la finalidad de malversar fondos. En otras palabras, la corrupción estimularía ciertas inversiones, que afectarían positivamente el desarrollo. Así, al interactuar con la sofisticación de la estructura productiva, los efectos del aumento del control de la corrupción, en el desarrollo se invertirían.

En el caso de los resultados encontrados en las variables rezagadas del cuadro 5, la estructura de producción más sofisticada parece mitigar los efectos de la corrupción pues acentuó los beneficios de su control. Este resultado es consistente con Ruhashyankiko y Yehoue (2006). En la literatura también se sugiere que la malversación de fondos produce resultados ineficientes para el desarrollo, conforme los argumentos de Rose-Ackerman (1997) y Mauro (1997b y 1998).

La amplitud de este tema puede entenderse a partir de la observación realizada por Hausmann y otros (2011) sobre las relaciones de la estructura productiva sofisticada. A pesar de sugerir una relación positiva entre el índice de complejidad económica y el PIB per cápita, como se puede observar en el gráfico 1, Hausmann y otros (2011) explican que dos países con un ingreso per cápita similar pueden presentar una complejidad económica diferente, como en el caso de China y el Perú. Mientras el PIB per cápita de China fue de 6.032,62 dólares en 2014 y el del Perú fue de 5.861,41 dólares (Banco Mundial (s/fa), en valores constantes de 2010), su índice de complejidad económica fue de 0,74 y -0,43, respectivamente. Si bien los autores utilizan este índice, el argumento también es válido cuando se utiliza el PIB sectorial para representar la estructura productiva.

Así, se busca entender por qué los efectos directos del control de la corrupción en el desarrollo son tan sensibles al considerar diferentes especificaciones. Esto puede obedecer a que el efecto de la corrupción puede depender de otros aspectos relativos a cada país. De ese modo, se espera que la corrupción sea no lineal y que la representación de sus efectos tenga forma de $U$.

La función cuadrática del control de la corrupción se estimó conforme la especificación representada por la ecuación (3) ${ }^{9}$.

$$
\begin{aligned}
& \operatorname{sub}_{t}=5,0271+0,8788 \mathrm{sub}_{t-1}-6,9247 \text { corcon }_{t}+9,2571 \operatorname{corcon}_{t}^{2}-0,0191 \text { fdii }_{t}-1,0149 \mathrm{hc}_{t} \\
& (0,8436)^{* * *}(0,0165)^{* * *} \quad(1,1867)^{* * *} \quad(1,4777)^{* * *} \quad(0,0103)^{*} \quad(0,1701)^{* * *}
\end{aligned}
$$

Al diferenciar en relación con el control de la corrupción observada, se constata que el control de la corrupción tiene un punto de inflexión con un valor igual a 0,374. En el gráfico 2 se muestra la relación cuadrática del control de la corrupción con el desarrollo (simulaciones de valores en una escala de 0 a 10 para la subalimentación).

En el gráfico 2 se puede observar que, a medida que aumenta el control de la corrupción, disminuye la subalimentación y que, a partir del punto de inflexión, un mayor control de la corrupción pasa a aumentar la subalimentación. Esto es consistente con Houston (2007), Sobral (2014) y Swaleheen (2011), el último de los cuales justifica ese resultado mediante el argumento de "lubricación de las ruedas del crecimiento económico".

\footnotetext{
9 Error estándar entre paréntesis. Los coeficientes de la ecuación son estadísticamente significativos a ${ }^{\star \star \star} p<0,01$ y ${ }^{\star} p<0,1$. Número de instrumentos: 80. Prueba de Sargan (Prob>chi2): 0,5827. Prueba de Arellano-Bond (autocorrelación "AR(2)"): 0,8578. Número de observaciones: 934. Número de países: 78.
} 


\section{Gráfico 2}

Efecto del control de la corrupción en el desarrollo

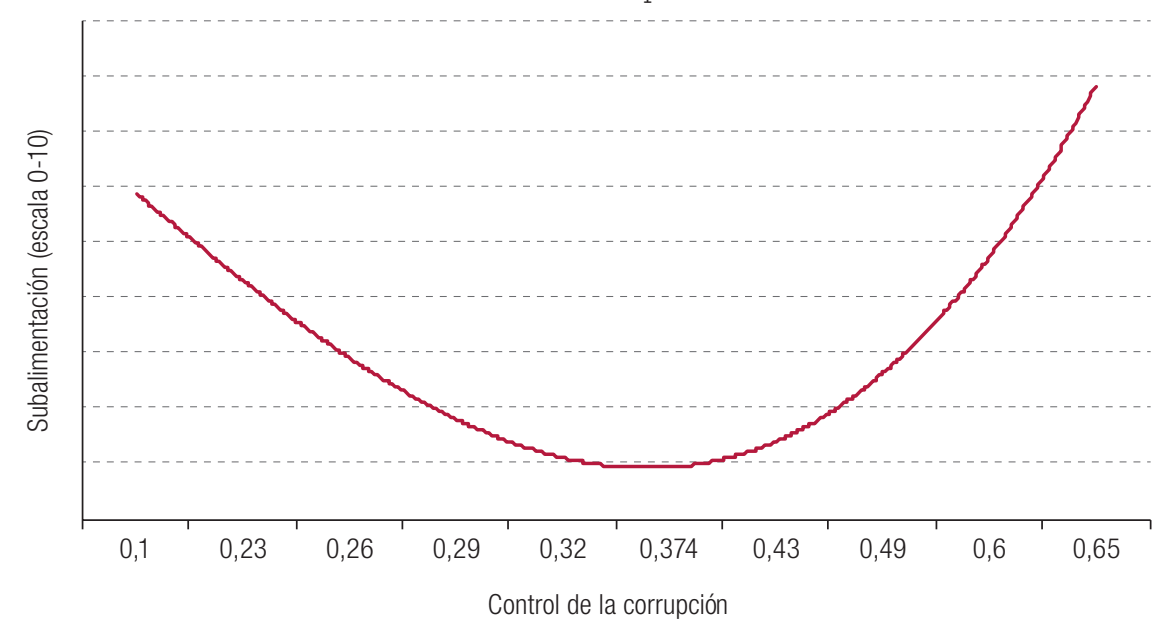

Fuente: Elaboración propia.

Al examinar los datos de control de la corrupción utilizados, relativos a 2014, se observa que el $61 \%$ de los 98 países de la muestra presenta un nivel de control de la corrupción de hasta 0,33, mientras el 39\% restante presenta un nivel de 0,42 en adelante. Esto significa que, en este contexto, la mayoría de los países de la muestra se puede beneficiar de un mayor control de la corrupción.

El análisis conjunto del desarrollo, la estructura productiva y la corrupción en un modelo aplicado permitió verificar el comportamiento no lineal de la relación y observar la manera en que se producen los efectos de la corrupción en el desarrollo por medio de la sofisticación de la estructura productiva, contribuyendo a la literatura existente sobre el tema en general.

\section{Consideraciones finales}

La literatura sobre el desarrollo es bastante amplia. La corrupción y la sofisticación de la estructura productiva del país son dos de los aspectos que se analizan entre los determinantes del desarrollo. A pesar de la creciente literatura relacionada con el tema, todavía existen lagunas, sobre todo en lo que se refiere a los efectos concomitantes de los aspectos de la corrupción y de la sofisticación de la estructura productiva. En consecuencia, cobran importancia las investigaciones que analizan la interacción entre las variables que se sabe inciden en el desarrollo, a fin de observar la intensidad de sus efectos.

Así, el objetivo de esta investigación consistía en analizar la manera en que la estructura productiva, la corrupción y los posibles efectos de la interacción entre esas dos variables influyen en el desarrollo socioeconómico de los países en desarrollo.

Si bien se constató que los efectos de la corrupción en el desarrollo de un país pueden ser positivos o negativos, hay indicios de un consenso acerca de su influencia negativa. Asimismo, se observó que la corrupción puede potenciarse en ambientes caracterizados por un mayor nivel tecnológico, porque las tecnologías más avanzadas en el proceso y la estructura de producción pueden facilitar la malversación de fondos.

Se buscó explorar los efectos de la corrupción en el desarrollo en dos ámbitos: en el primero el desarrollo está representado por el porcentaje de personas subalimentadas, mientras en el segundo la variable de desarrollo está representada por el PIB per cápita. A pesar de que ambas estimaciones 
difieren en cuanto a los efectos directos de la sofisticación de la estructura productiva en el desarrollo, sugieren que, cuando esa variable interactúa con el control de la corrupción, la estructura productiva más sofisticada tiende a potenciar los efectos de la corrupción en el desarrollo. Este resultado es importante a la hora de formular políticas económicas para fomentar el desarrollo de los países.

En cuanto a los efectos directos del control de la corrupción, no hubo estabilidad de los parámetros frente a los cambios en las especificaciones. La sensibilidad de los resultados en relación con el desarrollo socioeconómico se entendió como un indicio de un comportamiento no lineal y, por lo tanto, debe tratarse con cuidado cuando haya políticas anticorrupción. Con respecto a las variables de control, la variable capital humano resultó positiva para el desarrollo socioeconómico en diferentes estimaciones. Las variables inversión extranjera y agua potable resultaron sensibles a la especificación.

La contribución de este estudio consiste en analizar los efectos simultáneos de esos indicadores, ampliando la discusión sobre el tema. Además, el conjunto de variables seleccionadas permitió destacar los efectos de la corrupción y de la estructura productiva, tanto desde el punto de vista del desarrollo económico, como desde la perspectiva del desarrollo social.

Al constatar los efectos del control de la corrupción en el desarrollo ante diferentes estructuras productivas y la no linealidad de los efectos de la corrupción, se subraya la necesidad de considerar ambos aspectos al implementar políticas públicas, tanto orientadas a la sofisticación de la estructura de producción como a las políticas anticorrupción.

\section{Bibliografía}

Acemoglu, D. y T. Verdier (1998), "Property rights, corruption and the allocation of talent: a general equilibrium approach", The Economic Journal, vol. 108, № 450, Hoboken, Wiley.

Aidt, T., J. Dutta y V. Sena (2008), "Governance regimes, corruption and growth: theory and evidence", Journal of Comparative Economics, vol. 36, № 2, Amsterdam, Elsevier.

Akçay, S. (2006), "Corruption and human development", Cato Journal, vol. 26, № 1, Washington, D.C., Instituto Cato.

Arellano, M. y S. Bond (1991), "Some tests of specification for panel data: Monte Carlo evidence and an application to employment equations", Review of Economic Studies, vol. 58, № 2, Oxford, Oxford University Press.

Arellano, M. y O. Bover (1995), "Another look at the instrumental variable estimation of error-components models", Journal of Econometrics, vol. 68, № 1, Amsterdam, Elsevier.

Baltagi, B. H. (2005), Econometric Analysis of Panel Data, Chichester, John Wiley \& Sons.

Banco Mundial (s/fa), "World Bank open data" [en línea] http://data.worldbank.org.

(s/fb), "Worldwide Governance Indicators. International country risk guide" [en línea] info.worldbank.org/ governance/wgi/pdf/PRS.xlsx.

_ ( $\mathrm{s} / \mathrm{fc})$, "Worldwide Governance Indicators. WGl aggregation methodology" [en línea] http://info.worldbank. org/governance/wgi/Home/Documents\#wgiAggMethodology.

Bardhan, P. (1997), "Corruption and development: a review of issues", Journal of Economic Literature, vol. 35, №3, Nashville, Asociación Estadounidense de Economía.

Blackburn, K. (2012), "Corruption and development: explaining the evidence", The Manchester School, vol. 80, № 4, Hoboken, Wiley.

Blundell, R. y S. Bond (1998), "Initial conditions and moment restrictions in dynamic panel data models", Journal of Econometrics, vol. 87, № 1, Amsterdam, Elsevier.

Dalberto, C. R. (2016), "Corrupção, finanças políticas e liberdades econômicas: uma abordagem de dados em painel", ponencia presentada en el XIX Encuentro Nacional de Economía de la Región Sur, Florianópolis, Asociación Nacional de Centros de Posgrado en Economía (ANPEC) [en línea] https://www.anpec.org. br/sul/2016/submissao/files_l/i2-2c6ca123dd97082342dbfd8d08e1befc.pdf.

FAO (Organización de las Naciones Unidas para la Alimentación y la Agricultura) (2016), "Food security indicators" [en línea] http://www.fao.org/economic/ess/ess-fs/ess-fadata/en/\#.WKBDXW8rLIU. 
Feenstra, R. C., R. Inklaar y M. P. Timmer (2015), "The next generation of the Penn World Table", American Economic Review, vol. 105, N 10, Nashville, Asociación Estadounidense de Economía.

Foro Económico Mundial (2012), "Global Agenda Councils. Anti-corruption” [en línea] http://reports.weforum. org/global-agenda-council-2012/councils/anti-corruption.

Hartmann, D. y otros (2015), "Linking economic complexity, institutions and income inequality", Cornell University Library, Ithaca [en línea] https://arxiv.org/vc/arxiv/papers/1505/1505.07907v3.pdf.

Hausmann, R. y otros (2011), The Atlas of Economic Complexity: Mapping Paths to Prosperity, New Hampshire, Puritan Press [en línea] http://atlas.media.mit.edu/static/pdf/atlas/AtlasOfEconomicComplexity.pdf.

Hines, J. R. (1995). "Forbidden payment: foreing bribery and American business after 1977", NBER Working Paper, № 5266.

Houston, D. A. (2007), "Can corruption ever improve an economy?", Cato Journal, vol. 27, № 3, Washington, D.C., Instituto Cato.

Huntington, S. P. (2002), "Modernization and corruption", Political Corruption: concepts and contexts, A. J. Heidenheimer y M. Johnston (ed.), Nueva Jersey, Transaction Publishers.

Kaufmann, D., A. Kraay y M. Mastruzzi (2010), "The worldwide governance indicators: methodology and analytical issues”, World Bank Policy Research Working Paper, №5430, Washington, D.C., Banco Mundial.

Klitgaard, R. E. (1994), A corrupção sob controle, Río de Janeiro, Jorge Zahar.

Leff, N. H. (1964), "Economic development through bureaucratic corruption", American Behavioral Scientist, vol. 8, $\mathrm{N}^{\circ} 3$, Thousand Oaks, Sage.

Leite, C. y J. Weidmann (1999), "Does mother nature corrupt? Natural resources, corruption, and economic growth", IMF Working Paper, N WP/99/85, Washington, D.C., Fondo Monetario Internacional (FMI).

Li, H., L. C. Xu y H. Zou (2000), "Corruption, income distribution, and growth", Economics \& Politics, vol. 12, $\mathrm{N}^{\circ} 2$, Hoboken, Wiley.

Mahdavi, P. (2014), "Extortion in the oil states: nationalization, regulatory structure, and corruption", Political Science Department Working Paper, Los Angeles, Universidad de California [en línea] https://pdfs. semanticscholar.org/cf99/d839edac4d64e576553799fab2194798f798.pdf.

Mauro, P. (1998), "Corruption and the composition of government expenditure", Journal of Public Economics, $N^{\circ}$ 69, Amsterdam Elsevier.

(1997a), "The effects of corruption on growth, investment, and government expenditure: a cross-country analysis", Corruption and the Global Economy, K. A. Elliot (ed.), Washington, D.C., Instituto de Economía Internacional Peterson.

_ (1997b), "Why worry about corruption?", Economic Issues, N 6, Washington, D.C., Fondo Monetario Internacional (FMI).

(1995), "Corruption and growth", The Quarterly Journal of Economics, vol. 110, N 3, Oxford, Oxford University Press.

Naciones Unidas (s/fa), "Indicadores de los Objetivos de Desarrollo del Milenio" [en línea] http://mdgs.un.org/ unsd/mdg/Data.aspx.

(s/fb), "Indicadores de los Objetivos de Desarrollo del Milenio. Objetivo 1. Erradicar la pobreza extrema y el hambre" [en línea] http://mdgs.un.org/unsd/mdg/Metadata.aspx?Indicatorld=0\&Seriesld=566.

Nye, J. S. (1967), "Corruption and political development: a cost-benefit analysis", The American Political Science Review, vol. 61, N², Washington, D.C., American Political Science Association.

OCDE (Organización de Desarrollo y Cooperación Económicos) (2013), Issues Paper on Corruption and Economic Growth [en línea] http://www.oecd.org/g20/topics/anti-corruption/issues-paper-on-corruptionand-economic-growth.htm.

OEC (Observatorio de Complejidad Económica) (s/fa), "Economic complexity rankings", Cambridge, Massachusetts Institute of Technology (MIT) [en línea] https://oec.world/en/rankings/country/eci/. (s/fb), "How does complexity compare to GDP per capita? (2014)", Cambridge, Massachusetts Institute of Technology (MIT) [en línea] http://atlas.media.mit.edu/en/visualize/scatter/hs07/gdp_pc_constant/ show/all/all/2014. (s/fc), "What is economic complexity?", Cambridge, Massachusetts Institute of Technology (MIT) [en línea] http://atlas.media.mit.edu/en/resources/economic_complexity.

Rao, J. M. y J. M. Caballero (1990), "Agricultural performance and development strategy: retrospect and prospect", World Development, vol. 18, № 6, Amsterdam, Elsevier.

Reis, C. F. de B. (2012), "Estrutura produtiva e instituições no desenvolvimento econômico a partir de recursos naturais: uma análise teórica e crítica", Anais do XL Encontro Nacional de Economia, Porto de 
Galinhas, Pernambuco, Asociación Nacional de Centros de Posgrado en Economía (ANPEC) [en línea] https://www.anpec.org.br/encontro/2012/inscricao/files_l/i5-56cda81f3ee955d6769594f3830964cd.pdf.

Rock, M. T. y H. Bonnett (2004), "The comparative politics of corruption: accounting for the east Asian paradox in empirical studies of corruption, growth and investment”, World Development, vol. 32, № 6 , Amsterdam, Elsevier.

Rodrik, D. (2004), "Industrial policy for the twenty-first century" [en línea] https://www.sss.ias.edu/files/pdfs/ Rodrik/Research/industrial-policy-twenty-first-century.pdf.

Rose-Ackerman, S. (1997), "The political economy of corruption", Corruption and the Global Economy, K. A. Elliott (ed.), Washington, D.C., Instituto de Economía Internacional Peterson.

Ruhashyankiko, J. F. y E. B. Yehoue (2006), "Corruption and technology-induced private sector development", IMF Working Paper, N 06/198, Washington, D.C., Fondo Monetario Internacional (FMI).

Sachs, J. D. y A. M. Warner (2001), "The curse of natural resources", European Economic Review, vol. 45, $N^{\circ}$ 4-6, Amsterdam, Elsevier.

Schteingart, D. (2015), "Productive structure, composition of exports, technological capabilities and economic development: does what countries export absolutely matter?", ponencia presentada en la XIII Conferencia Internacional Globelics, La Habana, 23-25 septiembre.

Shleifer, A. y R. W. Vishny (1993), "Corruption", The Quarterly Journal of Economics, vol. 108, N 3, Oxford, Oxford University Press.

Sobral, E. F. M. (2014), "Corrupção e os seus efeitos sobre a dinâmica do crescimento econômico regional: uma análise do caso brasileiro”, tesis para optar al grado de magister, Recife, Universidad Federal de Pernambuco.

Sodré, F. R. A. (2014), "Os impactos da corrupção no desenvolvimento humano, desigualdade de renda e pobreza dos municípios brasileiros", tesis para optar al grado de magister, Recife, Universidad Federal de Pernambuco.

Swaleheen, M. (2011), "Economic growth with endogenous corruption: an empirical study", Public Choice, vol. 146, № 1-2, Berlín, Springer.

WITS (World Integrated Trade Solution) (2018), "Product group" [en línea] https://wits.worldbank.org/ CountryProfile/en/Country/BY-COUNTRY/StartYear/2002/EndYear/2014/TradeFlow/Export/Indicator/ XPRT-PRDCT-SHR/Partner/WLD/Product/OresMtls. 


\section{Anexo A1}

Cuadro A1.1

Listado de países

\begin{tabular}{|c|c|c|}
\hline $\begin{array}{l}\text { Albania } \\
\text { Angola } \\
\text { Arabia Saudita } \\
\text { Argelia } \\
\text { Argentina } \\
\text { Armenia } \\
\text { Azerbaiyán } \\
\text { Bahamas } \\
\text { Bahrein } \\
\text { Bangladesh } \\
\text { Belarús } \\
\text { Bolivia (Estado Plurinacional de) } \\
\text { Botswana } \\
\text { Brasil } \\
\text { Brunei Darussalam } \\
\text { Burkina Faso } \\
\text { Burundi } \\
\text { Camerún } \\
\text { Chile } \\
\text { China } \\
\text { Colombia } \\
\text { Congo } \\
\text { Costa Rica } \\
\text { Côte d'lvoire } \\
\text { Cuba } \\
\text { Ecuador } \\
\text { Egipto } \\
\text { El Salvador } \\
\text { Emiratos Árabes Unidos } \\
\text { Etiopía } \\
\text { Federación de Rusia } \\
\text { Filipinas } \\
\text { Gabón }\end{array}$ & $\begin{array}{l}\text { Gambia } \\
\text { Georgia } \\
\text { Ghana } \\
\text { Guatemala } \\
\text { Guinea } \\
\text { Guinea-Bissau } \\
\text { Guyana } \\
\text { Honduras } \\
\text { Hong Kong (Región Administrativa Especial de China) } \\
\text { India } \\
\text { Indonesia } \\
\text { Irán } \\
\text { Jamaica } \\
\text { Jordania } \\
\text { Kazajstán } \\
\text { Kenya } \\
\text { Kuwait } \\
\text { Líbano } \\
\text { Liberia } \\
\text { Libia } \\
\text { Madagascar } \\
\text { Malasia } \\
\text { Malawi } \\
\text { Malí } \\
\text { Marruecos } \\
\text { México } \\
\text { Mongolia } \\
\text { Mozambique } \\
\text { Namibia } \\
\text { Nicaragua } \\
\text { Níger } \\
\text { Nigeria } \\
\text { Omán }\end{array}$ & $\begin{array}{l}\text { Pakistán } \\
\text { Panamá } \\
\text { Papua Nueva Guinea } \\
\text { Paraguay } \\
\text { Perú } \\
\text { Qatar } \\
\text { República de Corea } \\
\text { República Democrática del Congo } \\
\text { República Dominicana } \\
\text { República de Moldova } \\
\text { República Unida de Tanzanía } \\
\text { Senegal } \\
\text { Serbia } \\
\text { Sierra Leona } \\
\text { Singapur } \\
\text { Sri Lanka } \\
\text { Sudáfrica } \\
\text { Sudán } \\
\text { Suriname } \\
\text { Tailandia } \\
\text { Togo } \\
\text { Trinidad y Tabago } \\
\text { Túnez } \\
\text { Turquía } \\
\text { Ucrania } \\
\text { Uganda } \\
\text { Uruguay } \\
\text { Venezuela (República Bolivariana de) } \\
\text { Viet Nam } \\
\text { Yemen } \\
\text { Zambia } \\
\text { Zimbabwe }\end{array}$ \\
\hline
\end{tabular}

Fuente: Elaboración propia.

Nota: Las estimaciones con la variable sub no incluyen los siguientes países: Bahamas, Bahrein, Burundi, Hong Kong (RAE de China), Libia, Papua Nueva Guinea, Qatar, República Democrática del Congo, Singapur y Sudán. 


\section{Anexo A2}

Cuadro A2.1

Estimaciones de control, subalimentación como variable dependiente, 2002-2014

\begin{tabular}{|c|c|c|c|c|}
\hline \multirow{2}{*}{ Variables } & \multicolumn{2}{|c|}{ Parciales } & \multicolumn{2}{|c|}{ Completas } \\
\hline & (B1) & (B2) & (B1) & (B2) \\
\hline \multirow{2}{*}{$\operatorname{sub}_{(t-1)}$} & $0,819^{\star \star \star}$ & $0,848^{\star \star \star}$ & $0,712^{\star \star \star}$ & $0,756^{\star \star \star}$ \\
\hline & $(0,0595)$ & $(0,0605)$ & $(0,0849)$ & $(0,0758)$ \\
\hline \multirow[t]{2}{*}{ controlcorrup } & $-3,238^{\star \star \star}$ & $-2,478^{*}$ & $-5,715^{\star \star \star}$ & 2,853 \\
\hline & $(1,155)$ & $(1,485)$ & $(2,094)$ & $(28,61)$ \\
\hline \multirow{2}{*}{$\operatorname{controlcorrup}_{(t-1)}$} & & 1,541 & & \\
\hline & & $(1,680)$ & & \\
\hline \multirow[t]{2}{*}{ coragro } & & & $0,328^{*}$ & \\
\hline & & & $(0,184)$ & \\
\hline \multirow{2}{*}{ coragro $_{(t-1)}$} & & & $-0,0154$ & \\
\hline & & & $(0,163)$ & \\
\hline \multirow[t]{2}{*}{ corser } & & & & $-0,0638$ \\
\hline & & & & $(0,500)$ \\
\hline \multirow{2}{*}{$\operatorname{corser}_{(t-1)}$} & & & & $-0,0784^{*}$ \\
\hline & & & & $(0,0440)$ \\
\hline \multirow[t]{2}{*}{ eci } & & $-0,623^{\star *}$ & & \\
\hline & & $(0,245)$ & & \\
\hline \multirow[t]{2}{*}{$e c i_{(t-1)}$} & & 0,0392 & & \\
\hline & & $(0,307)$ & & \\
\hline \multirow[t]{2}{*}{ gdpind } & & & 0,0434 & $0,138^{*}$ \\
\hline & & & $(0,0617)$ & $(0,0829)$ \\
\hline \multirow[t]{2}{*}{$\operatorname{gdpind}_{(t-1)}$} & & & $-0,0297$ & $-0,0568$ \\
\hline & & & $(0,0662)$ & $(0,0756)$ \\
\hline \multirow[t]{2}{*}{ gdpser } & & & & 0,117 \\
\hline & & & & $(0,147)$ \\
\hline \multirow[t]{2}{*}{ fdii } & & & $-0,0842^{*}$ & $-0,103$ \\
\hline & & & $(0,0510)$ & $(0,0655)$ \\
\hline \multirow[t]{2}{*}{$h c$} & & & $-4,740^{\star \star \star}$ & $-4,592^{\star \star \star}$ \\
\hline & & & $(1,475)$ & $(1,538)$ \\
\hline \multirow[t]{2}{*}{ gini } & $0,200^{\star \star \star}$ & 0,0169 & $-0,0191$ & $-0,0461$ \\
\hline & $(0,0566)$ & $(0,0184)$ & $(0,0349)$ & $(0,0416)$ \\
\hline \multirow[t]{2}{*}{ ores_metal } & $0,0206^{\star *}$ & $-0,0121^{\star \star \star}$ & $0,0499^{\star \star *}$ & $0,0490^{\star \star}$ \\
\hline & $(0,00865)$ & $(0,00416)$ & $(0,0176)$ & $(0,0192)$ \\
\hline \multirow[t]{2}{*}{ Constante } & $-6,173^{\star \star \star}$ & $0,904^{\star \star}$ & $16,56^{\star \star \star}$ & 9,029 \\
\hline & $(2,047)$ & $(0,437)$ & $(5,608)$ & $(7,388)$ \\
\hline Número de instrumentos & 33 & 17 & 31 & 21 \\
\hline Prueba de Sargan (Prob>chi2) & 0,6692 & 0,5307 & 0,9903 & 0,8736 \\
\hline $\begin{array}{l}\text { Prueba de Arellano-Bond } \\
\text { (autocorrelación "AR(2)") }\end{array}$ & 0,1925 & 0,1928 & 0,5678 & 0,3358 \\
\hline Observaciones & 266 & 236 & 223 & 223 \\
\hline Número de países & 24 & 23 & 22 & 22 \\
\hline
\end{tabular}

Fuente: Elaboración propia.

Nota: Algunas estimaciones corresponden a un número menor de países debido a la no disponibilidad de datos sobre las variables explicativas. Para la variable gini, existen 24 países: Argentina, Armenia, Belarús, Bolivia (Estado Plurinacional de), Brasil, Colombia, Costa Rica, Ecuador, El Salvador, Federación de Rusia, Georgia, Honduras, Kazajstán, México, Panamá, Paraguay, Perú, República de Moldova, República Dominicana, Serbia, Tailandia, Turquía, Ucrania, Uruguay. Para las otras variables, faltan los siguientes países: variable sub, no incluye: Bahamas, Bahrein, Burundi, Hong Kong (RAE de China), Libia, Papua Nueva Guinea, Qatar, República Democrática del Congo, Singapur y Sudán; variables eci y corceci, Bahamas, Brunei Darussalam, Burundi, Gambia, Guinea-Bissau, Guyana, República Democrática del Congo, Sierra Leona, Suriname; variables gdpind, gdpagro, gdpser, corind, coragro y corser, Angola, Bahrein, Kuwait, Liberia, Libia; variable fdii, Cuba; variable cre, Cuba, Irán; variable tech, Angola, Guinea-Bissau, Liberia, Libia, República Democrática del Congo; variable agua, Brunei Darussalam, Hong Kong (RAE de China), Libia; variable hc, Azerbaiyán, Bahamas, Belarús, Cuba, Georgia, Guinea, Guinea-Bissau, Guyana, Líbano, Libia, Omán, Papua Nueva Guinea, Suriname; variable ores_metal, Liberia, República Democrática del Congo. Error estándar entre paréntesis ** $p<0,01,{ }^{\star *} p<0,05,{ }^{*} p<0,1$. 


\section{Anexo A3}

Cuadro A3.1

Estimaciones de control, PIB per cápita como variable dependiente, 2002-2014

\begin{tabular}{|c|c|c|c|c|}
\hline \multirow{2}{*}{ Variables } & \multicolumn{2}{|c|}{ Parciales } & \multicolumn{2}{|c|}{ Completas } \\
\hline & (C1) & (C1) & (C2) & (C3) \\
\hline \multirow[t]{2}{*}{$\operatorname{loggdp}(t-1)$} & $0,867^{\star \star \star}$ & $0,856^{* * *}$ & $0,963^{\star * *}$ & $0,931^{\star * *}$ \\
\hline & $(0,0497)$ & $(0,145)$ & $(0,0483)$ & $(0,0342)$ \\
\hline \multirow[t]{2}{*}{ controlcorrup } & $0,225^{\star}$ & 0,401 & $-2,153$ & $0,241^{\star \star}$ \\
\hline & $(0,125)$ & $(0,576)$ & $(1,387)$ & $(0,117)$ \\
\hline \multirow{2}{*}{ controlcorrup $_{(t-1)}$} & 0,120 & $0,591^{*}$ & & \\
\hline & $(0,177)$ & $(0,315)$ & & \\
\hline \multirow{2}{*}{ corceci } & & & & $-1,169^{*}$ \\
\hline & & & & $(0,627)$ \\
\hline \multirow[t]{2}{*}{ coragro } & & $-0,0335$ & & \\
\hline & & $(0,0481)$ & & \\
\hline \multirow{2}{*}{ coragro $_{(t-1)}$} & & $-0,0512^{\star \star \star}$ & & \\
\hline & & $(0,0192)$ & & \\
\hline \multirow[t]{2}{*}{ corser } & & & $0,0382^{*}$ & \\
\hline & & & $(0,0231)$ & \\
\hline \multirow{2}{*}{ eci } & 0,0203 & & & $0,411^{*}$ \\
\hline & $(0,0289)$ & & & $(0,224)$ \\
\hline \multirow{2}{*}{$e c i_{(t-1)}$} & $0,0633^{\star \star \star}$ & & & \\
\hline & $(0,0242)$ & & & \\
\hline \multirow[t]{2}{*}{ gdpind } & & $-0,00331$ & 0,00124 & \\
\hline & & $(0,0120)$ & $(0,00759)$ & \\
\hline \multirow{2}{*}{$\operatorname{gdpind}_{(t-1)}$} & & 0,00221 & & \\
\hline & & $(0,0122)$ & & \\
\hline \multirow{2}{*}{ gdpagro } & & 0,0188 & & \\
\hline & & $(0,0154)$ & & \\
\hline \multirow[t]{2}{*}{ gdpser } & & & $-0,0225^{\star *}$ & \\
\hline & & & $(0,0103)$ & \\
\hline \multirow{2}{*}{$\operatorname{gdpser}_{(t-1)}$} & & & $0,0118^{\star * \star}$ & \\
\hline & & & $(0,00439)$ & \\
\hline \multirow[t]{2}{*}{ fdii } & & $0,00562^{\star *}$ & $0,00228^{\star \star}$ & $0,00575^{*}$ \\
\hline & & $(0,00280)$ & $(0,00102)$ & $(0,00305)$ \\
\hline \multirow[t]{2}{*}{$h c$} & & 0,144 & $0,0761^{\star *}$ & 0,0822 \\
\hline & & $(0,189)$ & $(0,0368)$ & $(0,0782)$ \\
\hline \multirow[t]{2}{*}{ agua } & & & & 0,00170 \\
\hline & & & & $(0,00493)$ \\
\hline \multirow[t]{2}{*}{ abecom } & & $-0,00113$ & & \\
\hline & & $(0,00124)$ & & \\
\hline \multirow[t]{2}{*}{ gini } & $0,00349^{\star \star}$ & 0,00306 & $0,00231^{\star \star}$ & $0,00533^{\star *}$ \\
\hline & $(0,00146)$ & $(0,00484)$ & $(0,00118)$ & $(0,00235)$ \\
\hline ores_metal & $0,000980^{\star *}$ & $-0,00268$ & $-0,000259$ & $-0,000838$ \\
\hline & $(0,000438)$ & $(0,00213)$ & $(0,000519)$ & $(0,000751)$ \\
\hline Constante & $0,889^{\star \star \star}$ & 0,613 & 0,594 & $-0,0907$ \\
\hline & $(0,316)$ & $(1,263)$ & $(0,499)$ & $(0,612)$ \\
\hline Número de instrumentos & 27 & 29 & 30 & 20 \\
\hline Prueba de Sargan (Prob>chi2) & 0,9472 & 0,9950 & 0,8644 & 0,4568 \\
\hline $\begin{array}{l}\text { Prueba de Arellano-Bond } \\
\text { (autocorrelación "AR(2)") }\end{array}$ & 0,0199 & 0,1284 & 0,0961 & 0,5115 \\
\hline Observaciones & 247 & 237 & 234 & 213 \\
\hline Número de países & 23 & 22 & 22 & 22 \\
\hline
\end{tabular}

Fuente: Elaboración propia.

Nota: Algunas estimaciones corresponden a un número menor de países debido a la no disponibilidad de datos sobre las variables explicativas. Para la variable gini, existen 24 países: Argentina, Armenia, Belarús, Bolivia (Estado Plurinacional de), Brasil, Colombia, Costa Rica, Ecuador, El Salvador, Federación de Rusia, Georgia, Honduras, Kazajstán, México, Panamá, Paraguay, Perú, República de Moldova, República Dominicana, Serbia, Tailandia, Turquía, Úcrania, Uruguay. Para las otras variables, faltan los siguientes países: variable sub, no incluye: Bahamas, Bahrein, Burundi, Hong Kong (RAE de China), Libia, Papua Nueva Guinea, Qatar, República Democrática del Congo, Singapur y Sudán; variables eci y corceci, Bahamas, Brunei Darussalam, Burundi, Gambia, Guinea-Bissau, Guyana, República Democrática del Congo, Sierra Leona, Suriname; variables gdpind, gdpagro, gdpser, corind, coragro y corser, Angola, Bahrein, Kuwait, Liberia, Libia; variable fdii, Cuba; variable cre, Cuba, Irán; variable tech, Angola, Guinea-Bissau, Liberia, Libia, República Democrática del Congo; variable agua, Brunei Darussalam, Hong Kong (RAE de China), Libia; variable hc, Azerbaiyán, Bahamas, Belarús, Cuba, Georgia, Guinea, Guinea-Bissau, Guyana, Líbano, Libia, Omán, Papua Nueva Guinea, Suriname; variable ores_metal, Liberia, República Democrática del Congo. Error estándar entre paréntesis ${ }^{\star * \star} p<0,01,{ }^{\star *} p<0,05,{ }^{*} p<0,1$. 